\title{
LA PÊCHE AU SAUMON DANS LE MASSIF ARMORICAIN : ÉTAT DES LIEUX ET MESURE DE L'EFFORT DE PÊCHE'1
}

\author{
J. SALANIÉ (1), Y. SURRY (2), P. LE GOFFE (3)
}

(1) et (3) Département d'économie rurale et gestion, Agrocampus Rennes, 65, rue de Saint-Brieuc, CS 84215, 35042 Rennes cedex.

(2) Department of Economics, Swedish University of Agricultural Sciences, P.O. Box 7013, SE-750 07 Uppsala, Sweden.

Reçu le 20 janvier 2005

Accepté le 19 avril 2005

Received January 20, 2005

Accepted April 19, 2005

\section{RÉSUMÉ}

Cette étude présente les résultats d'une enquête téléphonique effectuée auprès des pêcheurs de saumon de l'Ouest armoricain pour la saison 2002. Nous avons réuni un échantillon de 828 pêcheurs. Les résultats obtenus permettent de caractériser la population des pêcheurs de saumon et d'identifier leur effort de pêche ainsi que ses composantes. Nous montrons notamment, que l'effort individuel de pêche est très influencé par le revenu du pêcheur et la distance qui sépare son domicile du site de pêche.

En outre, nos résultats nous ont permis de déterminer les caractéristiques de l'effort de pêche sur les 28 rivières à saumon de la zone étudiée. Ces rivières présentent des situations très variées. En particulier, nous avons pu comparer la qualité de la pêche sur ces rivières grâce à des indicateurs tels que le nombre de captures par unité d'effort et la congestion. Nous démontrons que certains parcours de pêche sont en situation de congestion, point rarement évoqué dans la littérature française sur la pêche de loisir. II s'agit notamment des rivières qui accueillent plus de 5000 sorties de pêche par an.

Une comparaison de nos résultats avec ceux d'autres travaux permet de valider les réponses obtenues. Nos résultats sont cohérents et montrent que les enquêtes téléphoniques peuvent être un moyen efficace d'étude de la pêche de loisir en France.

Mots-clés : effort de pêche, enquête halieutique, qualité de la pêche, saumon.

\section{SALMON ANGLING IN WESTERN FRANCE: OVERVIEW AND MEASURE OF FISHING EFFORT}

\begin{abstract}
This paper presents the results of a phone survey concerning salmon anglers in Western France for year 2002. We collected data over a sample of 828 salmon anglers. Data were used to characterize salmon anglers'population and to identify fishing effort and

\footnotetext{
${ }^{1}$ Cette recherche a bénéficié du soutien financier de la région Bretagne dans le cadre du programme BREIZH. Les auteurs remercient Y. LEON pour ses commentaires. Les erreurs et omissions restent de la seule responsabilité des auteurs.
} 
its components. More specifically, we show that individual fishing effort is linked to their income and distance from their home to fishing sites.

Moreover, our results were used to estimate fishing effort characteristics for the 28 rivers of the study area. These rivers are very heterogeneous. We have been able to compare these situations using quality indicators like catch rate and congestion. We show that some fishing spots are overcrowded. This aspect of salmon angling has never been studied in France so far. The overcrowded rivers are those for which the total annual fishing effort exceeds 5000 visits.

The comparison of our results to previous French studies validates our survey results. Then, we believe that phone surveys can be an effective tool to evaluate recreational fishing in France.

Key_words: fishing effort, angling survey, angling quality, salmon.

\section{INTRODUCTION}

En France, les pêcheurs de saumon doivent déclarer leurs captures. Pour cela, on leur fournit un « assortiment » lorsqu'ils acquittent la taxe «salmonidés migrateurs ». Cet assortiment est généralement composé d'une fiche d'identification de la capture (lieu, poids, taille, etc.) et d'une pochette destinée à envoyer une écaille prélevée sur la capture au centre national d'interprétation des captures du Conseil Supérieur de la Pêche. Ce système est fondamental pour la gestion des stocks, car il permet de connaître le nombre de captures faites en France ainsi que la composition des stocks de saumon sur nos rivières par classes d'âge, de taille et de poids.

Cependant, si cet outil est utile pour la gestion des stocks, il est peu informatif pour la gestion de la pêche de loisir. II existe d'ailleurs très peu de données sur la quantité et les répartitions spatiale et temporelle de l'effort de pêche. Les seules données disponibles sont celles contenues dans les carnets de pêche tenus par des volontaires, une soixantaine chaque année, et certaines enquêtes ponctuelles menées généralement par les associations de pêche, le Conseil Supérieur de la Pêche (CSP) ou l'Institut National de la Recherche Agronomique (INRA).

Pourtant, la pêche de loisir, et en particulier celle des espèces « nobles » comme la truite ou le saumon, est reconnue pour l'importance de ses valeurs économiques d'usage et patrimoniale. Sa contribution aux économies régionales est substantielle et constitue un atout touristique pour des régions comme la Bretagne ou la Normandie (BONNIEUX, 2000). D'autre part, on constate que la pêche de loisir fait face à de nombreuses difficultés. Le nombre de pêcheurs de loisir est en baisse constante depuis une quinzaine d'années, au moins sur le domaine géré par les associations de pêche. Pour la pêche au saumon, la réglementation ne cesse d'évoluer ${ }^{2}$ sans apporter de réponses à ces problèmes. Ce constat d'échec s'explique en partie par une gestion qui ne cherche pas la maximisation du bien-être collectif. En particulier, les politiques de limitation de l'effort (fermetures hebdomadaires, recul de l'ouverture) minorent la richesse tirée par les pêcheurs de leur loisir (LE GOFFE et SALANIÉ, 2005). En parallèle, ces mesures ne permettent pas de répondre aux problèmes de congestion des parcours de pêche. Ce travail s'inscrit donc dans une étude complète de la demande de pêche au saumon dont il constitue la première étape.

\footnotetext{
${ }^{2}$ Quotas individuels, puis par rivières, variation du montant de la taxe, etc.
} 
Cet article présente les résultats d'une enquête menée auprès des pêcheurs de saumon de l'Ouest armoricain (Bretagne et Manche) pour la saison 2002. II vise à : Manche),

- caractériser l'effort de pêche sur les rivières de l'Ouest armoricain (Bretagne et

- identifier les facteurs influençant cet effort,

- et caractériser la population de pêcheurs et le rapport qu'ils ont à leur loisir.

Dans la première partie de l'article, nous présentons la méthodologie d'enquête utilisée. Les deux parties suivantes présentent les principaux résultats tirés de cette enquête, à savoir les principales caractéristiques des pêcheurs (deuxième partie) et un état des lieux des rivières à saumon dans la zone d'étude (troisième partie). Dans la quatrième partie, nous réalisons une agrégation de l'effort de pêche pour les rivières de la zone d'étude. Enfin, nous discutons nos résultats et les comparons aux données existantes dans la cinquième partie. Nous concluons en résumant les principaux apports de cette étude sur la pêche du saumon et en montrant leur intérêt dans l'analyse de l'effort de pêche et de la congestion. Mais avant toutes choses, nous souhaitons définir, en encadré I, certains termes propres à la description des données pour la pêche au saumon.

\section{ENCADRÉ I. DÉFINITIONS}

\section{BOX I. DEFINITIONS}

Bécard : Saumon de « descente » en migration vers la mer.

Capture : Tout poisson pris et sorti de l'eau. Une capture peut faire l'objet d'une remise à l'eau, même si ce n'est pas le cas général.

Castillons ou saumons d'automne: Saumons n'ayant passé qu'un seul hiver en mer. Ils remontent généralement pendant la saison d'été-automne.

Captures par unité d'effort (CPUE): Ratio reliant les captures à l'effort de pêche. Les CPUE seront exprimés en nombre de captures par sortie de pêche. L'inverse permet d'obtenir le nombre moyen de sorties à réaliser pour capturer un saumon (CPUE $=$ captures/sorties).

Effort de pêche: Nombre de sorties de pêche effectuées par le pêcheur au cours de la saison.

Ouest armoricain: Zone d'étude comprenant la région Bretagne et le département de la Manche.

Saison de pêche: Pour le saumon, on distingue en général deux saisons de pêche au cours de l'année calendaire : la saison de printemps et la saison d'automne. En général, l'ouverture de la pêche au saumon est fixée en mars et la fermeture en septembre. Cependant, il existe des modulations car pour chaque rivière, ces dates sont fixées par arrêtés préfectoraux.

Saumons de printemps : Saumons ayant passé plusieurs hivers en mer. On les capture généralement pendant la saison de printemps.

Sortie de pêche: Unité récréative de base. Elle correspond à la visite proprement dite et inclut le déplacement du pêcheur vers un site, puis le retour à son lieu de résidence. Généralement, les pêcheurs font une seule sortie par jour et celle-ci ne s'étend pas sur plus d'une journée. Nous ferons l'hypothèse que c'est le cas pour toutes les sorties de pêche au saumon recensées dans notre échantillon. 


\section{MÉTHODOLOGIE D'ENQUÊTE}

\section{Constitution de l'échantillon de pêcheurs}

Près de 2600 individus pratiquent chaque année la pêche au saumon en France. La pêche au saumon nécessite l'acquittement d'une taxe spéciale dite "salmonidés migrateurs". Elle autorise la pêche en rivière du saumon atlantique et de la truite de mer. Les ventes de taxes "salmonidés migrateurs " s'effectuent principalement dans les départements possédant des rivières à salmonidés migrateurs. Les principaux départements concernés sont la Manche, l'llle-et-Vilaine, les Côtes-d'Armor, le Morbihan, le Finistère et les Pyrénées-Atlantiques. Au total, 1900 des 2600 pêcheurs de saumon en France sont originaires d'un des quatre départements bretons ou du département de la Manche.

Cette population est particulièrement faible, comparée à la population nationale (moins de 0,005\%), ce qui rend quasiment impossible tout échantillonnage aléatoire au sein de la population française. La plupart des fédérations de pêche ne tiennent pas de fichiers de pêcheurs de saumon, et les bordereaux récapitulatifs de l'achat des cartes de pêche sont généralement inexploitables. En l'absence de fichiers d'adhérents, l'obtention de l'identité des pêcheurs est une difficulté majeure pour la conduite d'enquêtes. Néanmoins, les pêcheurs ayant effectué une capture de saumon durant la saison sont obligés de la déclarer. Pour constituer notre base d'échantillonnage, nous avons donc utilisé la base de données du CSP recensant les déclarations de captures de saumon. Pour chaque saison de pêche, cette base recense tous les pêcheurs ayant effectué au moins une capture, à l'exception, évidemment, des fraudeurs. Le taux de non-déclaration des captures est d'ailleurs estimé à 30-40 \% par le CSP (PREVOST et PORCHER, 1996 ; CSP, 1996).

L'utilisation de ces données comme base de sondage pose un problème d'autosélection identique à celui identifié par HECKMAN (1979). Les pêcheurs qui vont le plus souvent à la pêche ont plus de chance de réaliser au moins une capture et donc d'être échantillonnés. Pour limiter ce biais, nous avons constitué notre base d'échantillonnage à partir des trois dernières années de déclaration (i.e. 2000, 2001 et 2002). Ainsi une grande partie du problème d'auto-sélection devrait être éliminée. Néanmoins, des pêcheurs particulièrement peu efficaces, parce qu'ils manquent d'expérience ou parce qu'ils font très peu de sorties, peuvent ne pas avoir été échantillonnés.

Notre base de sondage contient donc tous les pêcheurs de saumon ayant pêché dans l'Ouest armoricain, à l'exception de ceux qui n'ont pas fait au moins une capture lors des trois dernières années sur une des rivières de la zone d'étude.

Étant donnée l'étendue géographique de la zone qui nous intéresse, les méthodes d'enquête sur site n'ont pas été retenues. Nous avons opté pour une enquête téléphonique moins coûteuse, et dont les taux de réponses sont généralement plus élevés que ceux d'une enquête postale (BILOCQ, 1996). II n'a pas été possible de mettre en place une stratégie d'échantillonnage fondée sur la méthode des quotas, car peu de statistiques fiables existent sur les pêcheurs de saumon. Cependant, nous avons établi des objectifs départementaux de sondage proportionnels aux effectifs départementaux de pêcheurs de saumon sur la base de 1000 pêcheurs enquêtés. Les résultats de l'enquête, en termes d'effectifs sondés ${ }^{3}$, sont présentés dans le tableau I.

\footnotetext{
${ }^{3} \mathrm{Au}$ total, 1166 individus ont été contactés. 206 d'entre eux n'étaient pas pêcheurs de saumon et 40 n'avaient pas pêché en 2002. Sur les 920 pêcheurs de saumon contactés en 2002, nous avons eu 89 refus, soit un taux de réponse de 90,3\%. Trois questionnaires étaient inexploitables, réduisant l'échantillon final à 828 pêcheurs. La base de sondage collectée ne contenait généralement ni adresses, à l'exception souvent de la commune de résidence, ni numéros de téléphone. Nous avons trouvé ces informations dans les «Pages Blanches » des annuaires téléphoniques de chaque département.
} 


\section{Tableau I}

Comparaison de la répartition géographique de la population de pêcheurs de saumon et de l'échantillon obtenu pour la saison 2002.

Table I

Comparison of the geographic distribution between salmon anglers population and our sample for year 2002.

\begin{tabular}{|l|c|c|c|c|}
\hline \multirow{2}{*}{$\begin{array}{c}\text { Département } \\
\text { County }\end{array}$} & \multicolumn{2}{c|}{$\begin{array}{c}\text { Population dans l'Ouest } \\
\text { armoricain } \\
\text { Population in Western France }\end{array}$} & \multicolumn{2}{c|}{$\begin{array}{c}\text { Population sondée } \\
\text { Sampled population }\end{array}$} \\
\cline { 2 - 5 } & effectifs & $\%$ & effectifs & $\%$ \\
\hline Ille-et-Vilaine & 127 & 5,9 & 68 & 8,18 \\
\hline Côtes d'Armor & 300 & 14,0 & 121 & 14,56 \\
\hline Finistère & 730 & 34,0 & 315 & 37,91 \\
\hline Morbihan & $\sim 200$ & 9,4 & 88 & 10,59 \\
\hline Manche & 638 & 29,7 & 199 & 23,95 \\
\hline $\begin{array}{l}\text { Autres départements } \\
\text { (total France) }\end{array}$ & $\begin{array}{c}\sim 150^{*} \\
(-700)\end{array}$ & 7,0 & 37 & 4,81 \\
\hline $\begin{array}{l}\text { TOTAL } \\
\text { (total France) }\end{array}$ & $\begin{array}{c}\sim \mathbf{2 ~ 1 5 0} \\
(-2 ~ \mathbf{~ 6 0 0 )}\end{array}$ & $\mathbf{1 0 0}$ & $\mathbf{8 2 8}$ & $\mathbf{1 0 0}$ \\
\hline
\end{tabular}

* Ce chiffre est retenu arbitrairement pour exclure les pêcheurs du Sud-Ouest pêchant sur les Gaves et des Nives et qui ne font pas de sorties sur les rivières du Grand Ouest à cause de la distance.

La structure géographique de notre échantillon est très proche et représentative de celle de la population de pêcheurs de saumon de l'Ouest armoricain. Nous avons soumis à enquête près de $44 \%$ de la population de pêcheurs de saumon fréquentant les rivières de la zone géographique concernée. On constate dans l'échantillon une légère sousreprésentation des pêcheurs de la Manche et une sur-représentation des pêcheurs du Finistère et de l'llle-et-Vilaine. Nous n'avons cependant procédé à aucun ajustement, car ces différences ne nous ont pas paru significatives.

\section{Procédure d'enquête}

L'enquête téléphonique a été menée en février et mars 2003, soit avant l'ouverture de la saison 2003. Les pêcheurs ont été interrogés sur la saison de pêche de l'année précédente. Les appels ont été réalisés systématiquement avec relance. Tous les pêcheurs figurant dans la base ont été appelés jusqu'à obtention des objectifs. Les informations obtenues concernent la saison 2002. Elles regroupent des données individuelles, ainsi que des informations de comportement désagrégées par rivière fréquentée et/ou par période de pêche.

Les pêcheurs ont été enquêtés sur leur fréquentation des rivières de l'Ouest armoricain. Parmi celles-ci, trois rivières n'étaient fréquentées par aucun pêcheur de notre échantillon : Le Dourduff, le Faou et le Guindy. La figure 1 identifie les rivières citées par les pêcheurs.

Dans ce travail, nous présentons parfois des statistiques croisant les caractéristiques socioprofessionnelles du pêcheur, la rivière fréquentée et la saison de pêche (saison de printemps ou d'été-automne). Ainsi un individu visitant trois rivières (deux au printemps et deux à l'automne, dont une rivière commune aux deux périodes) compte pour quatre observations. Pour ces statistiques, tout se passe comme si l'on avait quatre individus. Elles représentent les statistiques que l'on obtiendrait en faisant des enquêtes sur site. Nous obtenons ainsi des totaux supérieurs à 828 pêcheurs : ils se montent à 1174 pêcheurs de printemps par rivière et à 819 pêcheurs d'automne par rivière. 


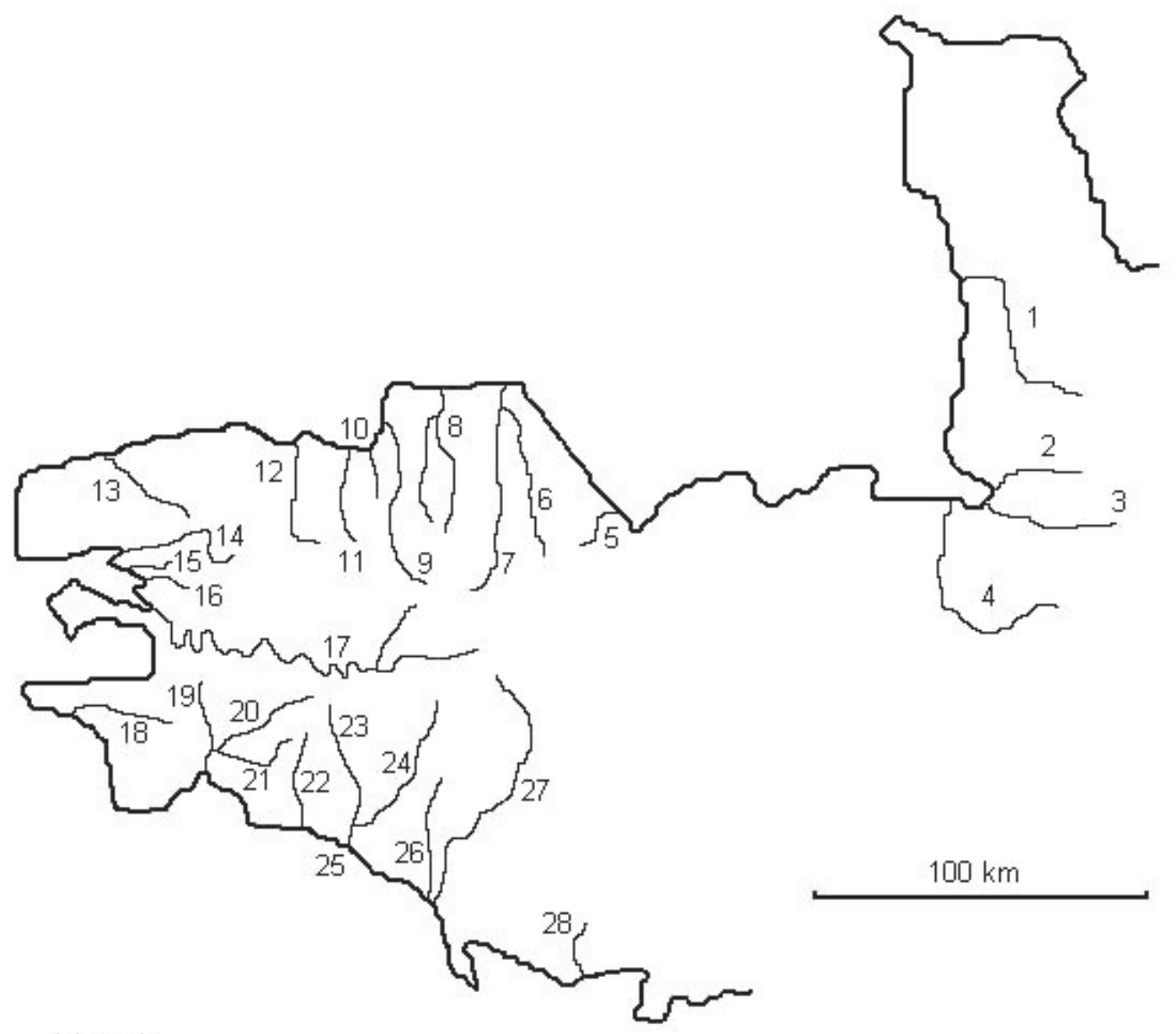

Légende

1. Sienne

6. Leff

11. Douron

16. Camfrout

21. Odet

26. Scorff

2. Sée

7. Trieux

12. Penzé

17. Aulne

22. Aven

27. Blavet

3. Sélune

8. Jaudy

13. Aber wrac'h

18. Goyen

23. Isole

4. Couesnon

9. Léguer

14. Elorn

19. Steïr

24. Ellé

5. Gouet

10. Yar

15. Mignonne

20. Jet

25. Laïta

\section{Figure 1}

Zone d'étude.

\section{Figure 1}

Study area.

\section{Les sources de biais dans les enquêtes}

Deux principales sources de biais dans les enquêtes téléphoniques sont identifiées par LYLE et al. (2002), le biais de mémoire et le biais de prestige.

Le biais de mémoire est lié à l'éloignement entre la date d'enquête et la période d'occurrence des événements ciblés. Les informations fournies par l'enquêté sont d'autant plus imprécises que l'événement est lointain et peu marquant. Ce problème est plus important pour les enquêtes portant sur une saison de pêche entière que sur la (ou les) dernière(s) sortie(s) de pêche. LYLE et al. (2002) préconisent l'emploi de carnets de suivi de l'activité du pêcheur et d'appels réguliers. Cette méthodologie très lourde et très coûteuse n'a pas pu être mise en place. Pour limiter le biais de mémoire, nous avons opté pour une enquête avant l'ouverture de la saison de pêche (en février et mars) 2003, 
période à laquelle nous pensons que les pêcheurs se remémorent leur saison passée, en préparation de celle à venir. Dans notre cas, le biais de mémoire est principalement lié aux questions portant sur l'effort de pêche, les autres étant toujours d'actualité (revenu, motivations, etc.). Les pêcheurs se souviennent généralement bien des captures de saumon qu'ils ont réalisées, même sur plusieurs saisons passées. Les captures de saumon sont très marquantes car rares et très valorisées par les pêcheurs. En revanche, le biais de mémoire peut affecter les réponses portant sur le nombre de sorties et les sites visités. BILOCQ (1996) souligne qu'il est important de poser des questions multiples et de proposer des associations d'idées pour stimuler la mémoire de l'enquêté. Pour quantifier l'effort de pêche au saumon, nous avons donc retenu la stratégie suivante :

- On demande au pêcheur une quantification générale de son effort de pêche pour la saison 2002. Pour cela, on lui donne des repères (nombre de jours de pêche dans l'année) et on lui propose de réfléchir en termes de fréquence (nombre de fois par semaine, fréquence pendant les vacances et les week-ends, etc.).

- Puis, on demande au pêcheur d'identifier les rivières qu'il a fréquentées pendant la saison 2002.

- Enfin, on lui demande le nombre de sorties effectuées sur chacune de ces rivières.

Nous obtenons donc deux estimations de l'effort de pêche: une globale et une détaillée par rivière. Cette stratégie permet de limiter le biais de mémoire car le pêcheur doit d'abord réfléchir à son effort global, puis le détailler et l'ajuster. Nous pensons que le biais de mémoire est faible pour notre enquête ; cependant nous remarquons dans nos données le biais d'arrondi identifié par LYLE et al. (2002). Parce que les pêcheurs ne se souviennent pas parfaitement du nombre de leurs sorties, ils arrondissent leurs réponses. Ainsi, deux pêcheurs ayant effectué respectivement 97 et 102 sorties indiqueront tous les deux avoir fait 100 sorties. Dans notre échantillon, nous remarquons qu'à partir de 15 sorties par an, ce biais est présent et se traduit par une proportion très élevée de « nombres ronds » dans les réponses.

Le biais de prestige est lié à la surévaluation par le pêcheur de son taux de réussite. La relation entre l'enquêteur et le pêcheur est très importante pour limiter ce biais (LYLE et al., 2002). Il s'agit de bien faire comprendre au pêcheur les objectifs de l'étude et de bien lui signifier que le taux de « bredouilles » est généralement très élevé pour ce type de pêche et qu'il est important d'avoir des réponses fiables dans ce domaine. Les questions concernant les captures ont été présentées de cette façon et les réponses paraissent tout à fait représentatives de la réalité (cf. calculs de CPUE).

\section{LE PÊCHEUR DE SAUMON}

L'effort de pêche sur les rivières à saumon de l'Ouest armoricain est effectué à $95 \%$ par des individus originaires de cette zone (Tableau I). Plus de $60 \%$ d'entre eux habitent la Manche ou le Finistère. Ils effectuent plus de $65 \%$ de l'effort de pêche pour $63 \%$ des captures. Ces proportions sont valables aussi bien pour la saison de pêche au saumon de printemps que pour celle des castillons.

\section{Portrait du pêcheur de saumon type}

Les pêcheurs de saumon interrogés sont exclusivement des hommes, âgés en moyenne de 51 ans. $77 \%$ d'entre eux déclarent être les seuls pêcheurs de leur foyer, composé en moyenne de trois personnes. Pour les $23 \%$ restant, les autres pêcheurs du foyer sont les enfants, dans une proportion de $62 \%$, les conjoints à $17 \%$ et les autres parents à $14 \%$. 
Un tiers des personnes interrogées n'a pas de diplôme. Un autre tiers de la population interrogée possède un CAP ou un BEP. Le reste des pêcheurs déclare disposer d'un diplôme au moins équivalent au baccalauréat. Les diplômés de niveau « Bac +4 » au moins ne représentent que $8 \%$ d'entre eux.

Plus d'un tiers (35\%) des pêcheurs sont retraités. $22 \%$ d'entre eux sont des ouvriers et $20 \%$ des employés ou professions intermédiaires. $8 \%$ des pêcheurs exercent une profession libérale et $9 \%$ sont cadres dans une entreprise ou administration. Seuls $1 \%$ des pêcheurs sont chômeurs et $4 \%$ étudiants. Parmi les retraités, on trouve une répartition des anciennes professions semblable à celle des actifs.

Le revenu moyen des pêcheurs est de $2200 €$ nets par mois. Peu d'entre eux (3\%) déclarent un revenu inférieur à $750 €$ par mois. $20 \%$ des pêcheurs ont un revenu compris entre 750 et $1200 €, 23 \%$ ont un revenu compris entre 1200 et $1800 €$ par mois. Enfin, $10 \%$ des pêcheurs interrogés ont un revenu supérieur à $3800 €$ mensuels. En retenant un temps de travail de 160 heures mensuelles, le pêcheur moyen de notre échantillon a un salaire de $13,75 €$ nets par heure, soit exactement la statistique obtenue au niveau national pour la tranche d'âge des 51-55 ans (INSEE, 2001). Les pêcheurs de saumons sont relativement aisés par rapport à la population totale de l'Ouest armoricain (salaire net horaire compris entre 9 et $10 €$ ) mais cela s'explique principalement par l'âge moyen élevé des pêcheurs de saumon.

La figure 2 montre les résultats d'une analyse factorielle des correspondances (AFC) ${ }^{4}$ reliant, en actif, les variables socio-démographiques (en gras) et, en illustratif, les variables d'effort (en italique). Le premier axe factoriel est un axe d'âge, tandis que le deuxième est un axe de revenu, corrélé aux variables de formation et d'emploi. L'effort individuel de pêche exprimé en nombre de sorties est mal représenté sur ce plan factoriel, indiquant que la catégorie socioprofessionnelle et l'âge du pêcheur ne sont pas des facteurs déterminants de l'effort de pêche. Par ailleurs, les pêcheurs n'habitant ni la Bretagne ni la Manche ont des niveaux de formation et des revenus plus élevés que ceux de la région soumise à enquête, et en particulier de la Manche. On constate également que les pêcheurs les moins âgés et les plus aisés fréquentent plus de rivières que les autres, ce que l'on interprète comme une recherche de diversité des expériences récréatives plus marquée pour ces pêcheurs. Enfin, les individus les plus jeunes pensent être plutôt débutants dans la pêche au saumon, alors que les plus âgés estiment être des pêcheurs confirmés, ce qui est cohérent avec les données sur l'expérience en années, corrélée positivement avec l'âge.

\section{Effort individuel de pêche}

Le pêcheur moyen effectue 42 visites par an, qui se répartissent équitablement entre la saison de printemps et saison d'automne. II fréquente 1,6 rivières, au printemps comme à l'automne. Les 784 pêcheurs de saumon de printemps font en moyenne 26 sorties pendant la saison de printemps. Les 586 pêcheurs de castillons en font 25 pendant celle d'été-automne. Le tableau II montre que $75 \%$ des pêcheurs effectuent moins de 60 sorties par an. $13 \%$ des pêcheurs font plus de 100 sorties de pêche au saumon par an.

Les pêcheurs originaires des départements de l'Ouest armoricain effectuent plus de visites que les autres. $68 \%$ des pêcheurs dont le domicile se situe en dehors de la zone d'étude font moins de 20 sorties par an sur les rivières de l'Ouest armoricain (Tableau II). La distance qui sépare les sites de pêche de la résidence de ces pêcheurs est l'explication principale de leur moindre fréquentation.

\footnotetext{
${ }^{4}$ Les AFC ont été effectuées à l'aide du logiciel SPAD (LAMBERT et al., 1996).
} 


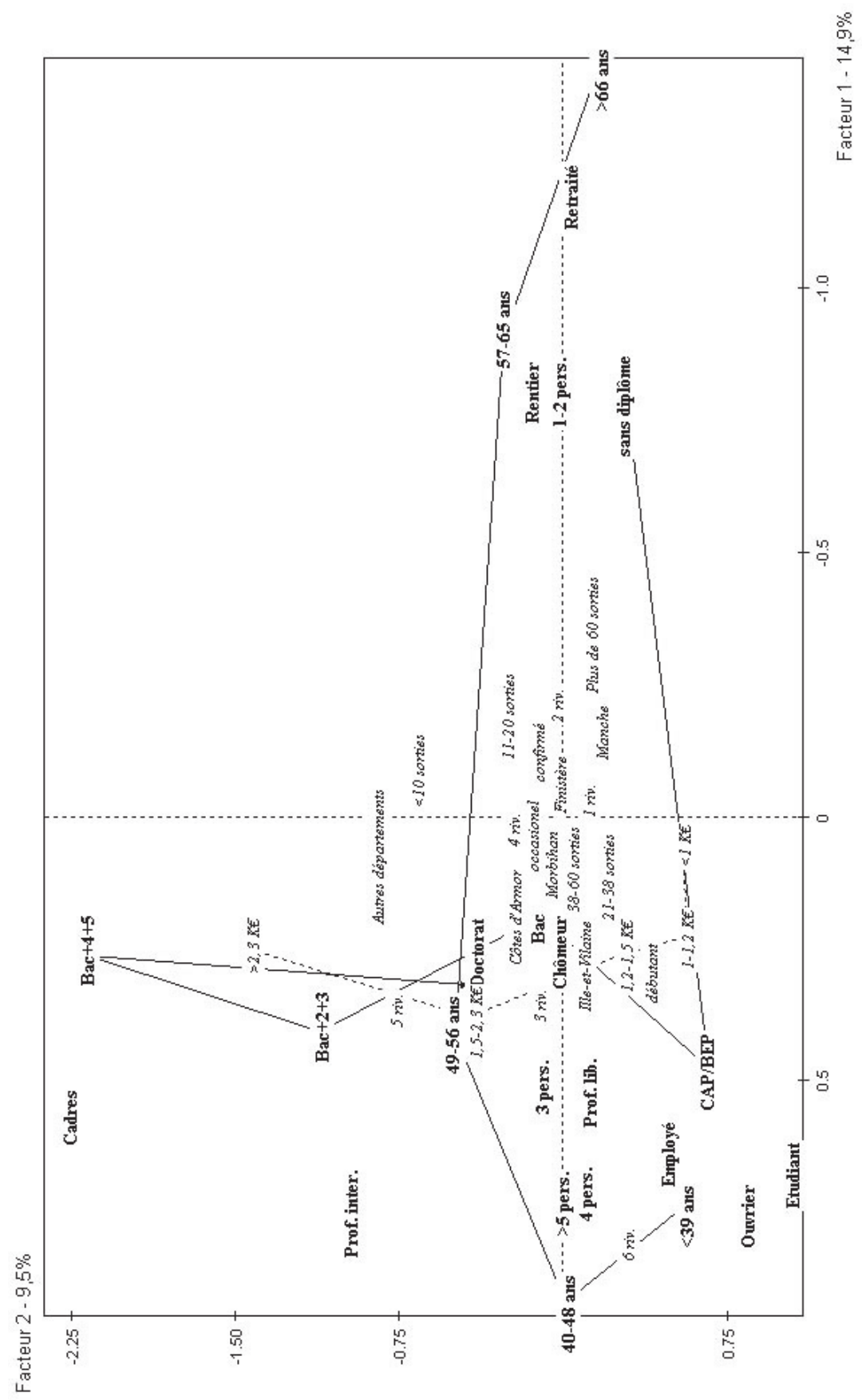

Légende: Variables actives en gras et variables illustratives en italique.

Figure 2

Projection de l'effort de pêche en fonction des caractéristiques sociodémographiques (premier plan factoriel).

Figure 2

Projection of fishing effort as a function of socio-demographic characteristics (first factorial plane). 


\section{Tableau II}

Nombre de pêcheurs par classe de visites et département d'origine.

Table II

Number of anglers per class of visits and per county of origin.

\begin{tabular}{|c|c|c|c|c|c|c|c|}
\hline $\begin{array}{c}\text { Classe de } \\
\text { visites } \\
\text { Classes of } \\
\text { visits } \\
\end{array}$ & $\begin{array}{l}\text { Côtes } \\
\text { d'Armor }\end{array}$ & Finistère & $\begin{array}{l}\text { Ille-et- } \\
\text { Vilaine }\end{array}$ & Manche & Morbihan & $\begin{array}{c}\text { Autres } \\
\text { Other } \\
\text { counties }\end{array}$ & Total \\
\hline $0-10$ & 17 & 55 & 15 & 30 & 11 & 13 & 141 \\
\hline $10-20$ & 13 & 62 & 14 & 26 & 16 & 12 & 143 \\
\hline $20-30$ & 23 & 38 & 5 & 22 & 11 & 6 & 105 \\
\hline $30-40$ & 20 & 41 & 14 & 23 & 12 & 1 & 111 \\
\hline $40-50$ & 4 & 11 & 3 & 13 & 8 & 2 & 41 \\
\hline $50-60$ & 12 & 34 & 4 & 28 & 4 & 3 & 85 \\
\hline $60-70$ & 9 & 13 & 5 & 9 & 6 & & 42 \\
\hline $70-80$ & & 5 & 2 & 2 & 3 & & 12 \\
\hline $80-90$ & 6 & 12 & 3 & 9 & 4 & & 34 \\
\hline $90-100$ & 2 & 2 & 1 & & 1 & & 6 \\
\hline $100-110$ & 8 & 22 & & 14 & 5 & & 49 \\
\hline $110-120$ & & 1 & & 4 & 1 & & 6 \\
\hline $120-130$ & & 2 & 2 & 2 & & & 6 \\
\hline $130-140$ & 1 & & & & & & 1 \\
\hline$>140$ & 6 & 17 & & 17 & 6 & & 46 \\
\hline Total & 121 & 315 & 68 & 199 & 88 & 37 & 828 \\
\hline
\end{tabular}

La figure 3 présente les résultats d'une analyse factorielle des correspondances reliant, en actif, les variables d'effort (en gras) et, en illustratif, les variables sociodémographiques (en italique). Le premier facteur est un axe d'effort. II oppose les pêcheurs qui parcourent des petites distances, mais font un grand nombre de visites, à ceux confrontés à de grandes distances et qui font peu de visites. On remarque un effet Guttman très prononcé (forme parabolique), classique en AFC, qui rend difficile I'interprétation du deuxième facteur (ESCOFIER et PAGES, 1998). L'interprétation du premier facteur est conforme à la théorie micro-économique de la demande. Du fait des distances élevées (qui s'interprètent ici comme un coût d'accès) qu'ils ont à parcourir pour atteindre les sites de pêche, seuls les pêcheurs ayant un consentement à payer supérieur au coût d'accès décident d'effectuer des sorties de pêche. Ce consentement à payer est supposé relié positivement au revenu. Dès lors, il s'exerce une sélection, sur la base du consentement à payer, entre les pêcheurs. Ainsi, seuls les pêcheurs les plus aisés sont prêts à parcourir de plus grandes distances, comme les pêcheurs résidant en Ille-et-Vilaine et en dehors de la zone étudiée.

Les sorties s'effectuent principalement à l'aval des rivières (42\%). Seuls $26 \%$ des pêcheurs pratiquent leur activité préférentiellement à l'amont des parcours de pêche. C'est une caractéristique bien connue de la pêche au saumon puisque seuls les saumons de montée sont autorisés à la capture, les bécards étant protégés. En général, les pêcheurs préfèrent explorer l'aval des rivières, où se trouvent généralement la majorité des saumons en cours de migration. $15 \%$ des pêcheurs déclarent connaître à peine les parcours qu'ils fréquentent, alors que plus de la moitié $(51,1 \%)$ déclarent très bien les connaître. 


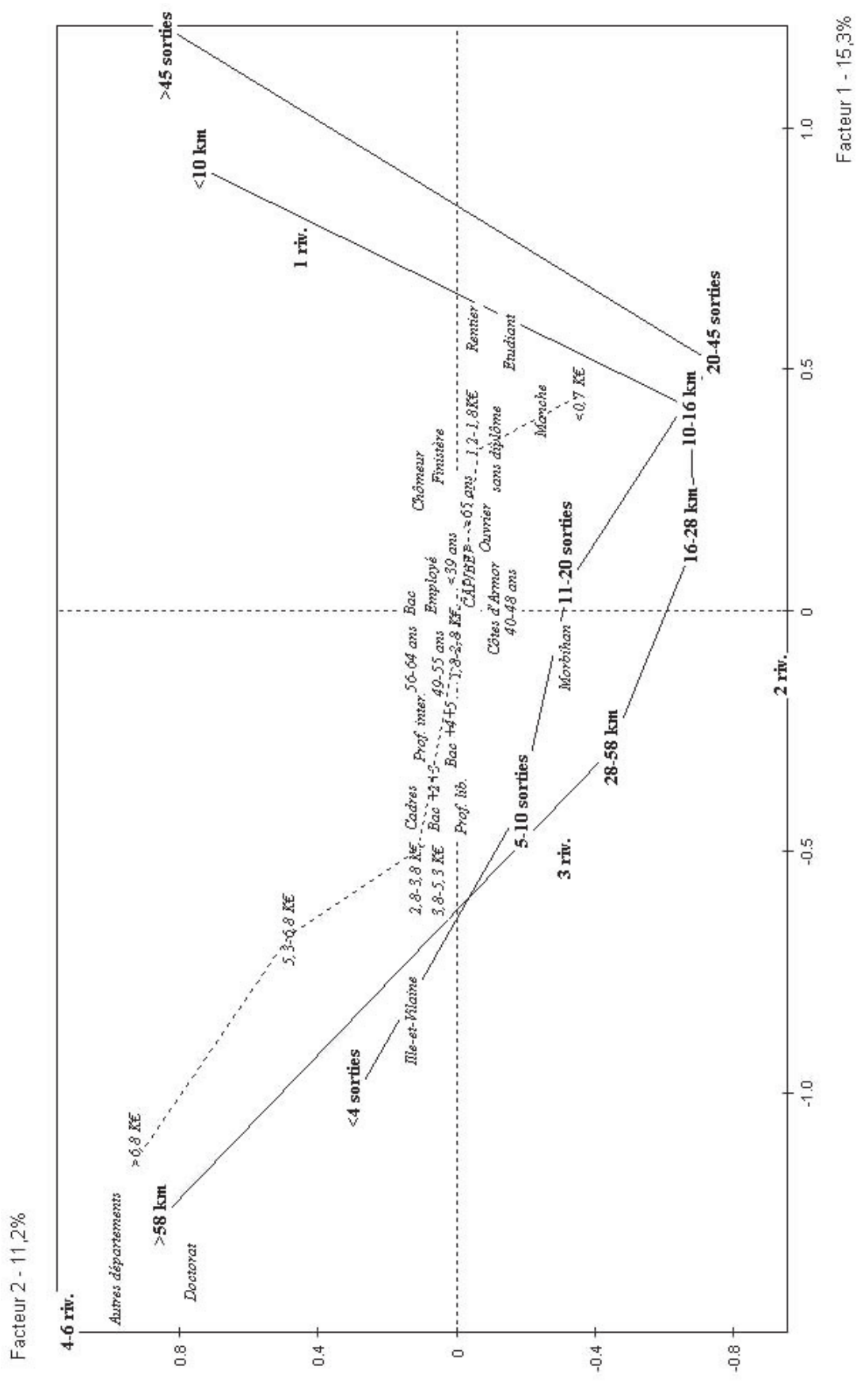

Légende: Variables actives en gras et variables illustratives en italique.

\section{Figure 3}

Projection des caractéristiques socio-démographiques en fonction de l'effort de pêche (premier plan factoriel).

\section{Figure 3}

Projection of socio-demographical characteristics as a function of fishing effort (first factorial plane). 
En moyenne, une sortie de pêche dure entre trois et cinq heures. Seul $1 \%$ des pêcheurs enquêtés déclare faire en moyenne des sorties d'une heure ou moins. II s'agit généralement des pêcheurs habitant à proximité des cours d'eau et qui pratiquent le « coup du soir ". Près d'un tiers $(30,9 \%)$ des pêcheurs font des sorties d'une durée supérieure à cinq heures. Les pêcheurs les plus jeunes font des sorties plus longues que les pêcheurs les plus âgés, ce qui est conforme aux résultats obtenus par BONNIEUX (2000), pour la Sée, la Sélune et l'Elorn.

Enfin, les deux tiers (63 \%) des pêcheurs pratiquent leur loisir plutôt seuls, le tiers restant préférant pêcher avec un groupe d'amis.

\section{Captures de saumon}

Le pêcheur moyen de notre échantillon réalise 1,06 captures par an. Le pêcheur de printemps moyen ne capture que 0,38 saumons de printemps par an, soit moins qu'à l'automne où ce nombre monte à 0,97 castillon par pêcheur et par an. Cette donnée est cependant très variable. On recense $66 \%$ de pêcheurs bredouilles sur l'année. Au maximum, un pêcheur prend chaque année six saumons de printemps et dix castillons.

Dans notre échantillon, la CPUE moyenne ${ }^{5}$ est de 0,025 captures par sortie. II faut donc en moyenne 40 sorties pour réaliser une capture. La CPUE est nettement plus faible au printemps $(0,015)$ qu'à l'automne $(0,040)$. Les principaux facteurs de variation de la CPUE individuelle sont l'expérience du pêcheur et l'appât qu'il utilise. Cependant, nous ne discutons pas les résultats concernant les appâts puisqu'ils reposent sur l'appât que les pêcheurs déclarent utiliser le plus souvent, pas nécessairement l'appât avec lequel ils ont effectivement effectué les captures.

L'expérience, exprimée en nombre d'années de pêche, influence la CPUE des pêcheurs selon une courbe en " $U$ " inversée (Figure 4). Les pêcheurs les moins expérimentés sont les moins efficaces. Les pêcheurs déclarant moins de cinq années d'expérience dans la pêche du saumon ont une CPUE de 0,013, alors que la moyenne de l'échantillon est de 0,025. Cette efficacité inférieure à la moyenne peut être expliquée par une moins bonne connaissance des techniques de pêche au saumon, des rivières, des bons coins de pêche et du comportement des saumons. Les pêcheurs dont l'expérience est inférieure à cinq années ont une CPUE inférieure à la moyenne quel que soit l'appât, aussi bien pour le saumon de printemps que pour les castillons. De même, les pêcheurs les plus expérimentés, qui sont également les plus âgés, ont une CPUE inférieure à la moyenne (0,021 pour les pêcheurs de plus de 45 ans d'expérience). Cette situation semble s'expliquer par le choix de l'appât. Les pêcheurs les plus âgés utilisent principalement les techniques de lancers, moins performantes que la mouche ou la crevette (CPUE de 0,020 contre 0,031 et 0,038 respectivement sur l'ensemble de l'échantillon). D'autre part, les pêcheurs les plus âgés font des sorties plus courtes, ce qui influence négativement leur CPUE. Le calcul de la CPUE exprimée en heures n'est pas possible de façon fiable pour notre échantillon, mais nous pensons qu'il permettrait de montrer une meilleure efficacité des pêcheurs plus âgés. Enfin, les pêcheurs qui ont entre 20 et 30 ans d'expérience sont les plus efficaces.

\footnotetext{
${ }^{5}$ Les calculs de CPUE présentés dans cette partie sont effectués à partir des effectifs totaux de l'échantillon. Par exemple, la CPUE des pêcheurs de la classe «10-15 ans d'expérience » est calculée comme la somme des captures faites par les pêcheurs de cette classe, divisée par la somme des visites qu'ils effectuent. Cette méthode de calcul ne nous permet ni de calculer des intervalles de confiance ni d'effectuer les tests statistiques habituels.
} 


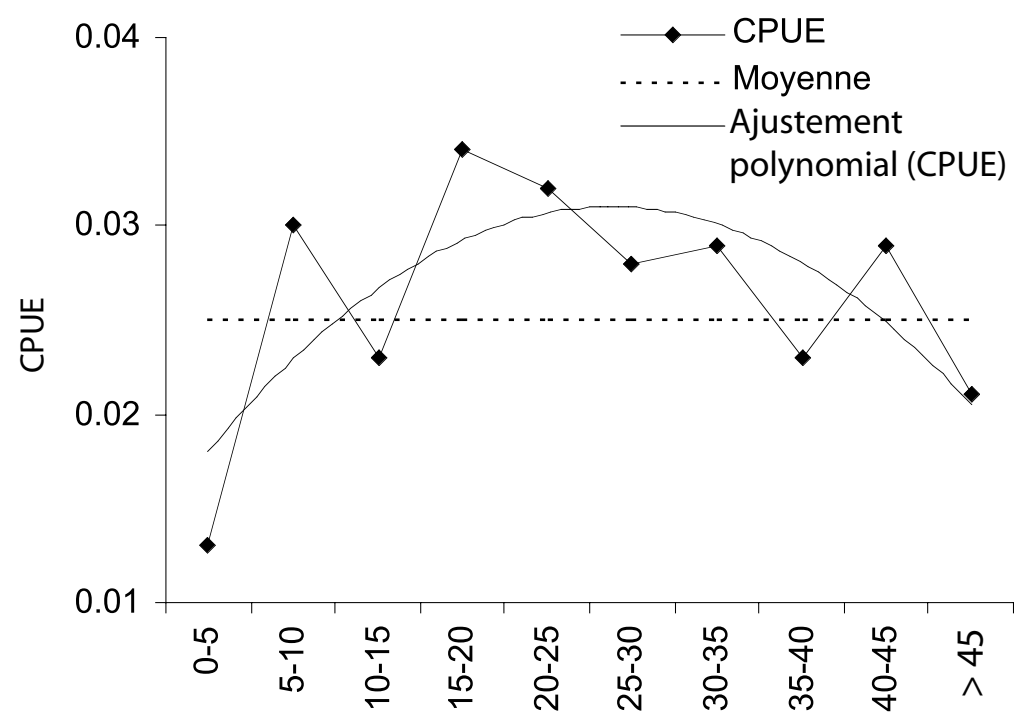

Classe d'expérience (années)

Note : la forme polynomiale retenue est quadratique, d'équation de régression $y=0,012+0,0067 x-0,0006 x^{2}$ et de coefficient de détermination $\left(R^{2}\right)$ égal à 0,53 .

\section{Figure 4}

Relation entre la CPUE et l'expérience de pêche.

\section{Figure 4}

Relationship between CPUE and angling experience.

\section{Les pêcheurs et leur loisir}

Dans les paragraphes qui suivent, nous nous intéressons aux relations qui lient les pêcheurs à leur loisir. Nous abordons tout d'abord leurs motivations pour la pratique de la pêche au saumon, puis les préférences qui influencent leurs choix de sites de pêche.

Le premier facteur de motivation des pêcheurs est la passion qu'ils ont pour cette activité. $73 \%$ des pêcheurs affirment qu'il s'agit d'un facteur très important dans leur pratique de cette activité de loisir, alors que seulement $2 \%$ déclarent que cela n'est pas important. Les possibilités d'admirer la nature et de se relaxer sont également des critères jugés très importants par les pêcheurs (63\% et $56 \%$ respectivement). La possibilité d'être seul est un critère important à très important pour $64 \%$ d'entre eux, alors que les $36 \%$ restants n'estiment pas cela important. Les réponses sont similaires pour la possibilité d'être en compagnie de sa famille ou de ses amis lorsque l'on va à la pêche. D'autre part, l'exercice physique effectué lors de la pratique de la pêche est un critère très important pour $23 \%$ des pêcheurs, alors qu'il ne l'est pas pour $19 \%$ d'entre eux. Enfin, $47 \%$ des pêcheurs déclarent que la possibilité de manger leur pêche n'est pas une raison pour qu'ils pratiquent cette activité de loisir. Ils sont $18 \%$ pour qui c'est une raison très importante.

Lorsqu'ils choisissent leur lieu de pêche, $57 \%$ des pêcheurs déclarent que la beauté de la rivière, et notamment son caractère sauvage, est un critère très important, voire décisif. II est important pour $37 \%$ d'entre eux. Seuls $6 \%$ des pêcheurs déclarent que c'est un critère qui influence peu le choix du site de pêche. Notons que la clarté de l'eau est un critère largement moins décisif puisque seuls $38 \%$ des personnes interrogées 
jugent ce critère très important, tandis que $25 \%$ le trouvent peu important. Les captures ne sont pas un facteur primordial dans la décision concernant le choix du site de pêche. Le nombre de captures qu'ils peuvent faire sur une rivière est peu important pour $60 \%$ des pêcheurs et très important pour seulement $10 \%$ des pêcheurs. II en va de même pour la taille des captures.

En outre, $13 \%$ des pêcheurs considèrent que la proximité par rapport à leur domicile est un critère très important dans leur choix du site de pêche. Dans le même temps, $47 \%$ des pêcheurs jugent ce critère peu important. Enfin, la fréquentation des parcours est un critère influent. Pour $24 \%$ des pêcheurs, la possibilité de pêcher sur des parcours peu fréquentés est un critère très important. Ce critère est majeur pour $44 \%$ des pêcheurs et importe peu pour $23 \%$ d'entre eux. Nous explorerons plus avant les questions de fréquentation des parcours dans le paragraphe consacré aux facteurs influençant la qualité de l'expérience récréative.

Les pêcheurs de saumon sont donc des passionnés. Si la majorité d'entre eux ne déclare pas attacher d'importance à l'abondance des captures, ils sont en revanche concernés par une pêche dans des rivières d'aspect sauvage et peu fréquentées. Nombreux sont les pêcheurs qui ne sont pas particulièrement intéressés par une pêche de proximité ; ils parcourent d'ailleurs en moyenne plus de kilomètres par sortie que les autres (Figure 5 et Annexe III). Ceux qui préfèrent les rivières à caractère sauvage parcourent également de plus grandes distances (Figure 5). En revanche, les préférences pour les parcours peu fréquentés sont sans lien apparent avec les distances parcourues.

\section{Appréciation par les pêcheurs de la qualité de la pêche et des mesures de gestion}

Les pêcheurs ont été interrogés sur l'effet de certains éléments limitant la qualité de la pêche de loisir. $83 \%$ des pêcheurs interrogés pensent que les pollutions agricoles ont un effet très néfaste sur la qualité de pêche. Parmi les différentes propositions, c'est l'élément jugé le plus négativement. Les captures en mer, faites par les pêcheurs professionnels, sont également perçues comme un facteur très négatif pour la qualité de la pêche de loisir pour $71 \%$ des pêcheurs. Pour $8 \%$ d'entre eux, les captures de saumons faites en mer n'influencent pas la qualité de la pêche en rivière. D'autre part, près de la moitié des pêcheurs pensent que la canalisation des rivières constitue un élément très limitant de la qualité de la pêche récréative. Enfin, $73 \%$ des pêcheurs pensent que les captures faites par les pêcheurs de loisir n'ont pas d'effet sur la qualité de leur loisir.

La fréquentation des parcours est également un élément qui joue sur la satisfaction retirée de la pêche lorsqu'elle aboutit à des situations de congestion. Nous avons demandé aux pêcheurs leur perception de la congestion ${ }^{6}$ sur les parcours qu'ils fréquentent en leur attribuant une note variant de 1 à 5 selon une échelle croissante allant de «très peu fréquenté » à « trop fréquenté ».

Sur les rivières étudiées, la note moyenne est de 3,22. Les parcours de pêche de l'Ouest armoricain sont jugés comme fortement fréquentés par $41 \%$ des pêcheurs (Tableau III). Mais les rivières connaissent des situations de congestion hétérogènes, comme nous le verrons dans la partie consacrée aux rivières.

\footnotetext{
${ }^{6}$ La question suivante portant sur la congestion a été posée aux pêcheurs enquêtés « Vous diriez que les zones de pêche que vous fréquentez sur la rivière " $X$ " sont plutôt ? : très peu (1), peu (2), moyennement (3), beaucoup (4) ou trop fréquentées (5) ».
} 
Tableau III

Répartition des réponses concernant l'appréciation de la congestion sur les parcours de pêche.

Table III

Distribution of the answers concerning congestion at fishing sites.

\begin{tabular}{|l|c|c|}
\hline \multicolumn{1}{|c|}{ Congestion } & $\begin{array}{c}\text { Effectifs } \\
\text { Numbers }\end{array}$ & $\%$ \\
\hline Très peu fréquenté / Very little congested & 91 & 6,97 \\
Peu fréquenté / Little congested & 233 & 17,85 \\
Moyennement fréquenté / Acceptable congestion & 445 & 34,10 \\
Très fréquenté / Very congested & 375 & 28,74 \\
Trop fréquenté / Highly congested & 161 & 12,34 \\
\hline Total & $\mathbf{1 3 0 5}$ & $\mathbf{1 0 0 , 0 0}$ \\
\hline
\end{tabular}

Les pêcheurs ont également été interrogés sur l'impact que les mesures de gestion ont sur la qualité de la pêche. Les totaux autorisés de captures (TAC), les repeuplements et les fermetures hebdomadaires sont jugés favorablement par $60 \%$ des personnes interrogées. Le recul de l'ouverture est une mesure perçue de façon plus mitigée. Ainsi, $37 \%$ d'entre eux estiment que le recul de l'ouverture a un effet positif sur la qualité de la pêche. Ils sont autant à penser le contraire et $26 \%$ jugent cette mesure sans effet. Pour les pêcheurs, les mesures de préservation des stocks de saumon (TAC, repeuplements et parcours «no-kill ») sont bénéfiques et permettent d'améliorer la qualité de la pêche. En revanche, les mesures qui induisent une limitation de l'effort de pêche sont mal perçues, à l'exception des fermetures hebdomadaires.

La figure 5 présente les résultats d'une AFC sur les préférences récréatives et la perception des mesures de gestion. Le premier facteur oppose les pêcheurs qui sont contre les mesures de restriction de l'effort de pêche (en positif) à ceux qui sont favorables (en négatif). Le deuxième facteur oppose les pêcheurs déclarant avoir des préférences pour les rivières sauvages et peu fréquentées (en négatif) et ceux pour qui ces caractéristiques ne sont pas importantes (en positif). Les pêcheurs opposés aux mesures de limitation de l'effort (TAC, fermetures hebdomadaires, recul de l'ouverture, etc.) sont ceux qui effectuent le plus de sorties et parcourent les plus petites distances, c'est-àdire des locaux. Ils ne sont également pas en faveur de l'Entente Halieutique du Grand Ouest (EHGO). Les pêcheurs de la Manche font partie de cette catégorie. A l'inverse, les pêcheurs de l'Ille-et-Vilaine et du Morbihan, sont plutôt réceptifs aux mesures de limitation de l'effort de pêche. Ces résultats sont à relier à l'éloignement de ces pêcheurs de la ressource. Les pêcheurs d'llle-et-Vilaine sont très nombreux à fréquenter les rivières de la Manche et des Côtes-d'Armor et ceux du Morbihan les rivières du Finistère (celles de Quimper et de Quimperlé). Les pêcheurs favorables à l'EHGO parcourent en moyenne une plus grande distance par sortie. Inversement, les pêcheurs de la Manche sont très proches de la ressource et perçoivent négativement la limitation de leur effort. Ils sont en revanche contre l'EHGO qui augmente potentiellement l'effort de pêche sur leurs rivières et détériore donc la qualité de la pêche sur ces dernières. On remarque d'ailleurs que les pêcheurs opposés à l'EHGO et au recul de l'ouverture sont des pêcheurs qui parcourent de petites distances pour se rendre à un site de pêche. On note également que ceux qui sont favorables aux parcours «no-kill » parcourent de plus grandes distances que les autres et font moins de sorties. 


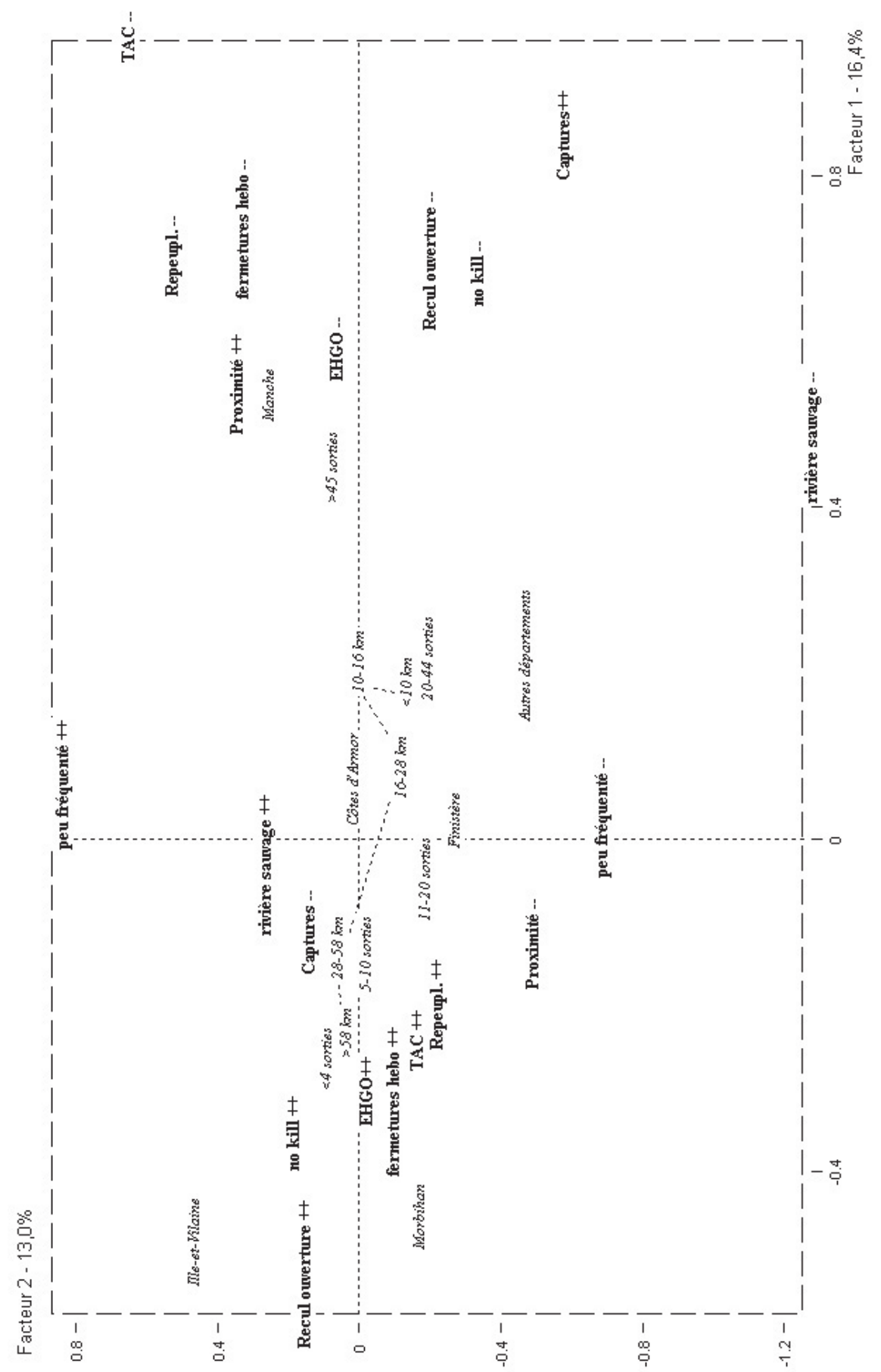

Légende: Variables actives en gras et variables illustratives en italique. ++ =favorable/intéressé par, - = défavorable/pas intéressé par.

Figure 5

Projection de l'effort de pêche en fonction de la perception des mesures de gestion par les pêcheurs (premier plan factoriel).

\section{Figure 5}

Projection of fishing effort as a function of individual perception of management (first factorial plane). 


\section{Les autres types de pêches pratiquées par les pêcheurs de saumon}

La plupart des pêcheurs de saumon pratiquent d'autres types de pêche. La pêche à la truite en rivière est pratiquée par $85 \%$ d'entre eux. Près de la moitié des pêcheurs de saumon pratiquent également la pêche en mer. $42 \%$ pêchent les carnassiers. En revanche, moins d'un pêcheur de saumon sur cinq pratique la pêche des poissons blancs ou la pêche de la truite en réservoir.

Les pêcheurs de saumon voyagent beaucoup, puisqu'un tiers d'entre eux ont été pêcher à l'étranger lors de cinq dernières années. Les voyages de pêche concernent très majoritairement les lles britanniques (57\%), l'Europe du Nord (14\%) et l'Amérique du Nord (9 \%). Certaines destinations comme l'Europe du Sud (7 \%), l'Europe de l'Est (4\%) et l'Afrique (4\%) sont également des destinations d'importance. Notons enfin que dans notre échantillon, les pêcheurs ont été sur tous les continents, mais certaines destinations comme l'Asie, la Russie ou l'Océanie restent marginales, étant visitées par moins de $1 \%$ des pêcheurs. $70 \%$ des séjours de pêche à l'étranger sont effectués pour pêcher le saumon. $18 \%$ des pêcheurs se rendent à l'étranger pour pratiquer la pêche à la truite. Les autres pêcheurs pratiquent la pêche au gros, la pêche aux carnassiers ou d'autres types de pêche (pêche sous-marine, etc.).

En France, les pêcheurs de saumon sont également 14 \% à fréquenter des parcours payant à la journée (réservoirs, etc.). Cette proportion est très proche de celle observée pour la truite en réservoir et il est possible que les parcours payants fréquentés par les pêcheurs de saumons soient les parcours de pêche à la truite en réservoir.

Les pêcheurs de saumon sont donc principalement des pêcheurs de salmonidés en eaux vives. Ils pêchent peu en lac ou en réservoir et, hormis les carnassiers, ils recherchent peu les espèces non salmonicoles. Toutefois, les pêcheurs de saumon sont également intéressés par la pêche en mer. De plus, un tiers des pêcheurs de saumon effectue des séjours de pêche à l'étranger. Là encore, les espèces salmonicoles sont de loin les plus recherchées.

II existe une forte discrimination entre les pêcheurs dans la pratique des autres pêches, et elle est principalement liée à un effet revenu. Les résultats d'une AFC sur la relation entre les caractéristiques socio-démographiques des pêcheurs (en actif), et les autres types de pêche pratiqués (en illustratif) sont présentés dans la figure 6 . II s'agit du même plan factoriel que celui utilisé dans la figure 2 où les deux premiers facteurs sont respectivement un axe d'âge et un axe de revenu. On remarque que les pêcheurs les plus aisés sont ceux qui vont à l'étranger, qui fréquentent des parcours payants à la journée, qui pêchent la truite en réservoir (ces parcours sont généralement payants) ou qui pratiquent la pêche en mer. Inversement, les pêcheurs les moins aisés pratiquent moins ces pêches et plutôt la pêche aux carnassiers ou aux poissons blancs.

\section{SITUATION DES RIVIÈRES À SAUMON DU MASSIF ARMORICAIN}

Dans cette partie, nous effectuons un état des lieux des diverses rivières à saumon dans l'Ouest armoricain en analysant les deux éléments suivants: l'effort de pêche propre à chaque rivière et leur qualité de pêche évaluée en termes de congestion et de CPUE. Pour ce faire, nous avons constitué des données agrégées pour chaque rivière qui apparaissent en annexe 1 .

\section{Effort de pêche par rivière}

L'effort de pêche est très différent selon les rivières étudiées. Les rivières historiquement les plus poissonneuses sont celles qui attirent le plus de pêcheurs (Figure 7), que ce soit pour la pêche au saumon de printemps ou au castillon. La grande 


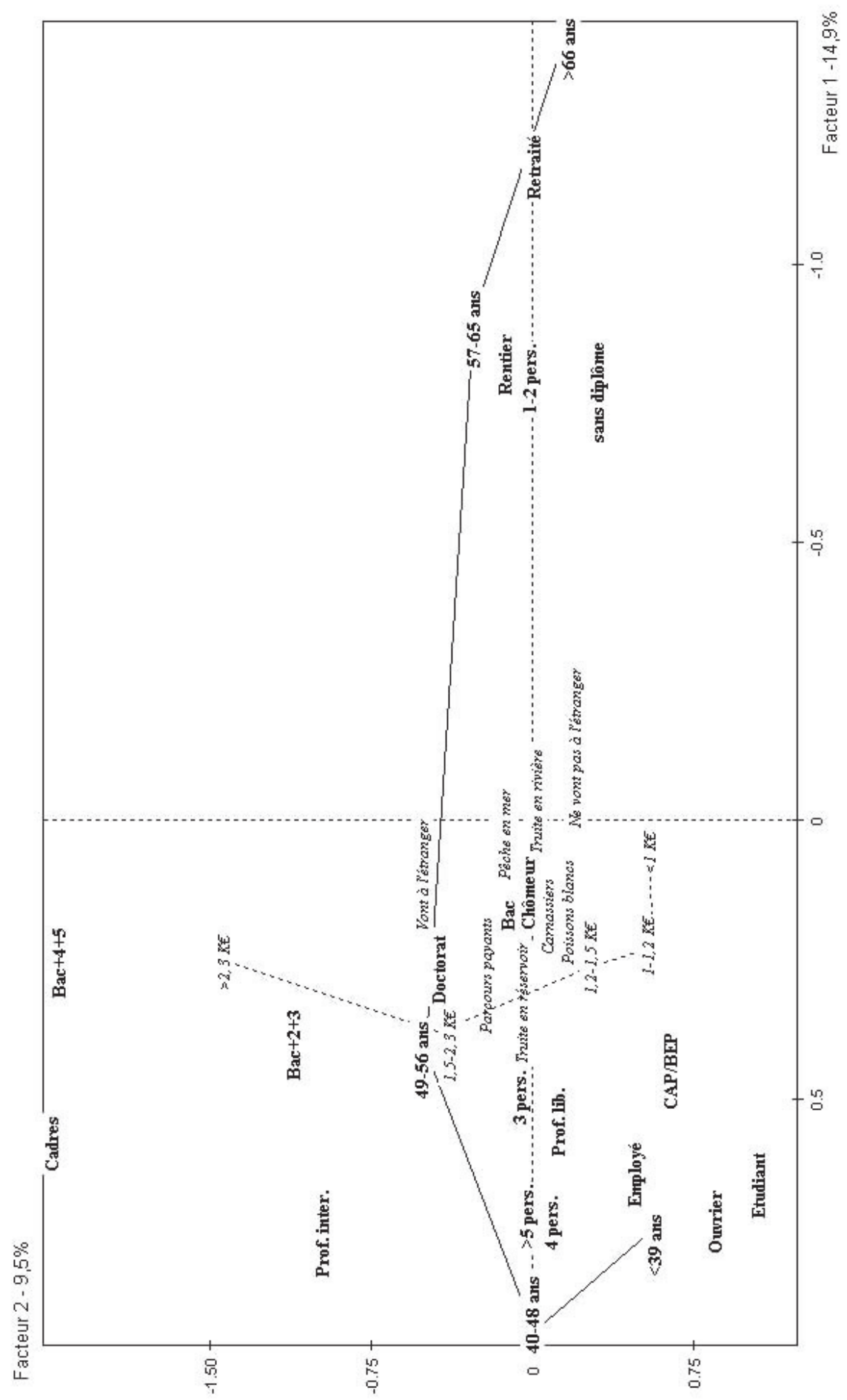

Légende : Variables actives en gras et variables illustratives en italique.

\section{Figure 6}

Projection des autres pêches pratiquées en fonction des caractéristiques sociodémographiques (premier plan factoriel).

\section{Figure 6}

Projection of other types of fishing as a function of socio-demographic characteristics (first factorial plane). 
majorité des visites est faite au printemps. Ceci est dû au différentiel qui existe en termes de pêcheurs pratiquants, car le nombre moyen de visites par pêcheurs est très proche pour les deux saisons. Les principales rivières en terme d'effort de pêche sont la Sée, la Sélune, l'Aulne, le Blavet, le Léguer et l'Ellé. A elles seules, ces six rivières représentent $65 \%$ de l'effort de pêche annuel total exprimé en nombre de visites. Si on ajoute l'Aven, l'Elorn, le Trieux et la Sienne, on constate alors que $80 \%$ de l'effort total est concentré sur un tiers des rivières.

Du point de vue de la saison, l'effort de pêche au printemps diffère nettement de celui de la saison d'été-automne. 784 (95\%) pêcheurs fréquentent les rivières au printemps pour seulement $586(71 \%)$ pêcheurs à l'automne. L'effort total annuel des pêcheurs de notre échantillon est de 35099 visites dont près de $60 \%$ effectuées au printemps.

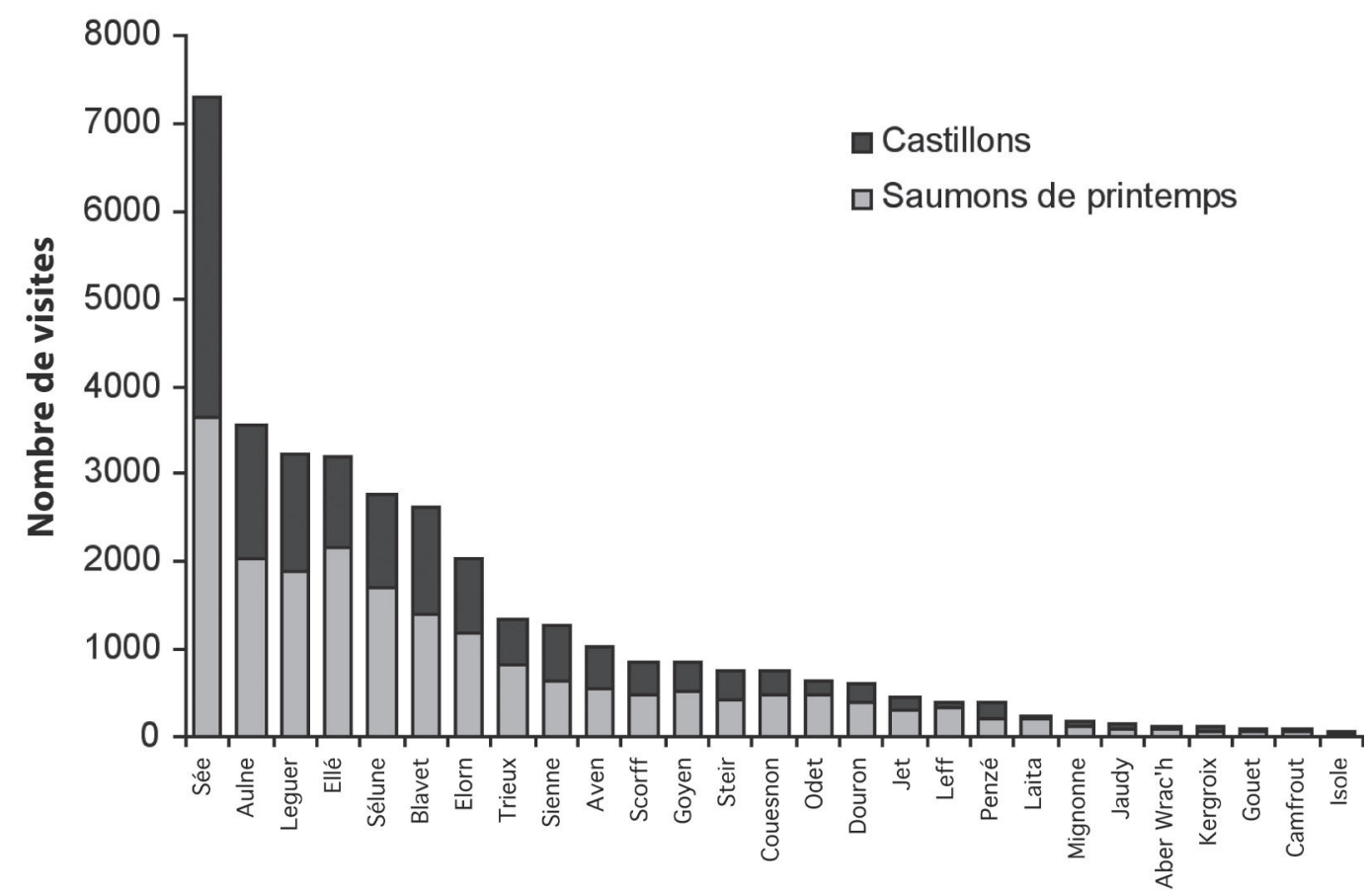

Figure 7

Effort total de pêche par rivière.

\section{Figure 7}

Total fishing effort per river.

Au total, les 35000 visites de l'échantillon ont occasionné près d'un million de kilomètres pour une distance moyenne de $27,8 \mathrm{~km}$ par sortie ${ }^{7}$. Les rivières les plus réputées font en moyenne l'objet de sorties plus longues, comme c'est le cas pour l'Aulne, l'Ellé, l'Elorn, la Sélune ou le Trieux (Annexe I). A l'inverse, les sorties les plus courtes

\footnotetext{
7 Les distances ont été calculées à l'aide du Système d'Information Géographique (SIG) de calcul d'itinéraires routiers Mappy (site internet http://www.mappy.com) entre le barycentre de la commune de résidence du pêcheur et le barycentre de la commune de destination sur la rivière. Nous remercions l'entreprise Mappy pour son soutien technique.
} 
sont effectuées sur de petites rivières comme le Jet, le Jaudy, le Camfrout ou le Goyen. Les pêcheurs font des sorties plus longues en moyenne pour la pêche au saumon de printemps $(30,2 \mathrm{~km})$ que pour la pêche au castillon $(24,4 \mathrm{~km})$. Nous pensons qu'il s'agit là d'un élément indiquant une plus forte valorisation des saumons de printemps. La pêche des castillons est une pêche plus locale.

L'éloignement moyen ${ }^{8}$ des pêcheurs aux rivières donne un aperçu de l'attractivité des rivières. On retrouve une situation très hétérogène. Les rivières les plus réputées attirent des pêcheurs plus éloignés, puisque l'éloignement moyen dépasse les $50 \mathrm{~km}$ pour l'Aulne, l'Elorn, l'Aven, le Léguer et la Sélune. Les rivières plus petites (Camfrout, Mignonne, Jaudy, Steïr par exemple) sont fréquentées par des pêcheurs locaux éloignés en moyenne de 15 à $20 \mathrm{~km}$ de la rivière. Sur l'Aulne, l'Aven et l'Elorn, on trouve des pêcheurs parcourant plus de $1000 \mathrm{~km}$. Pour venir pêcher sur le Blavet, le Léguer, la Penzé, l'Ellé, le Douron, l'Odet et le Scorff, certains pêcheurs parcours plus de $500 \mathrm{~km}$. Les petites rivières comme l'Aber Wrac'h, le Leff, le Jet, la Mignonne, le Steïr et le Jaudy n'attirent pas sur leur parcours de pêcheurs distants de plus de $50 \mathrm{~km}$.

\section{Des rivières aux situations de qualité contrastées}

La qualité de la pêche, appréciée par la note de congestion ou par la CPUE, varie fortement selon les rivières de la zone étudiée.

En matière de congestion des parcours de pêche, certaines rivières comme l'Odet, la Penzé, le Leff ou le Scorff sont jugées plutôt peu fréquentées (Figure 8). De fait, pour ces rivières, près de $60 \%$ des pêcheurs ont répondu «très peu " ou "peu fréquenté " (Annexe II). Quasiment aucun pêcheur n'a répondu «trop fréquenté » pour juger des parcours de pêche de ces rivières. Six rivières se situent au-dessus de la moyenne $(3,22)$ : La Sélune, la Sée, le Trieux, le Léguer, l'Aulne et le Douron. A l'exception du Douron, il s'agit également des rivières sur lesquelles l'effort de pêche est le plus élevé. La Sée, la Sélune et le Trieux obtiennent des notes moyennes de congestion supérieures à 3,5. Pour ces rivières, $20 \%$ des pêcheurs, ou plus, jugent qu'elles sont «trop fréquentées ". Pour la Sélune, ce sont $79 \%$ des pêcheurs qui estiment qu'elle est « très ou trop fréquentée ".

La figure 8 illustre la relation qui existe entre le nombre de visites estimées par an, ramené à la taille du parcours ${ }^{9}$ de pêche, appelée congestion "objective », et l'appréciation de la congestion faite par les pêcheurs, appelée congestion " subjective ". Plus la densité de pêcheurs le long d'une rivière est importante, plus la note moyenne de congestion de cette rivière augmente. La relation liant ces deux éléments est non linéaire et croissante. Du point de vue du pêcheur, l'effet de la fréquentation sur sa perception de la qualité de la pêche augmente plus vite que la densité réelle au site de pêche, pour les rivières dont la fréquentation est inférieure à 1 pêcheur par jour et par km "pêchable » de rivière ${ }^{10}$. Les premiers pêcheurs induisent plus de congestion que les suivants et des situations de congestion sont atteintes rapidement. L'effet de la congestion est à mettre en relation avec les motivations des pêcheurs pour leur loisir. La pêche est une activité pratiquée pour ce qu'elle apporte de calme, de solitude et de contact avec la nature, ce que ne peuvent pas procurer les parcours les plus densément fréquentés.

\footnotetext{
${ }^{8}$ Distance entre le site de pêche et le domicile du pêcheur. A la différence des calculs de distance, on ne tient pas compte du nombre de sorties effectuées.

${ }^{9}$ Pour les canaux (Aulne et Blavet), la longueur « pêchable » du parcours de pêche a été fixée arbitrairement à $20 \%$ de la longueur totale du parcours. On sait en effet que, sur ces rivières, les pêcheurs de saumon ne pêchent qu'en amont des écluses, sur les murets. Cet ajustement permet de tenir compte de la très forte densité de pêcheurs dans ces zones alors que le parcours de pêche n'est pas exploité dans sa totalité par les pêcheurs.

${ }^{10}$ La congestion « objective » a été ramenée au débit par sa racine carrée.
} 


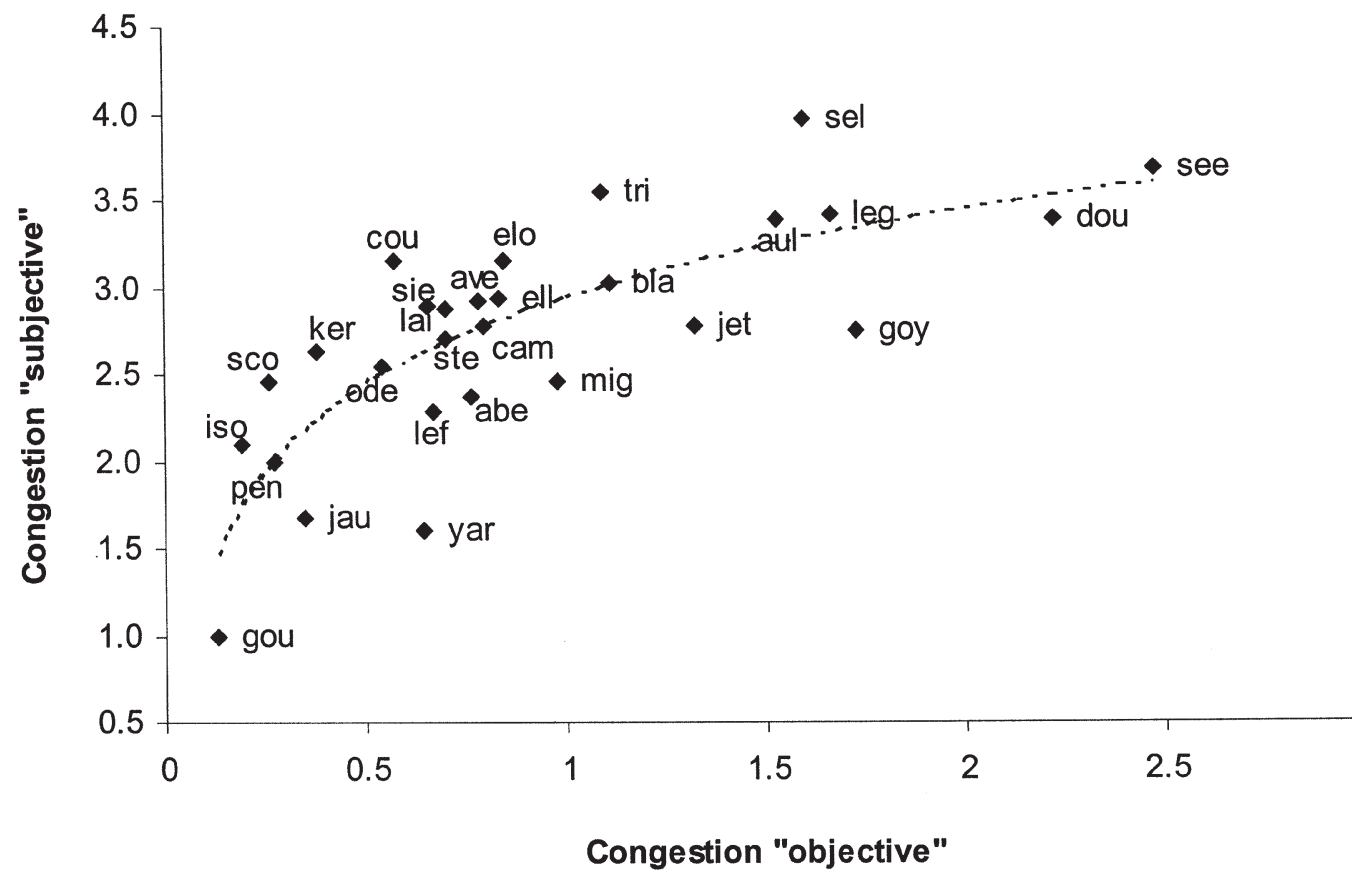

Légende : Les noms des rivières ont été abrégés aux trois premières lettres. La courbe d'ajustement figurant sur le graphique a pour équation $y=2,95+0,73 \ln (x)$ et pour coefficient de détermination $\left(R^{2}\right) 0,62$.

\section{Figure 8}

Relation entre la congestion et la densité de fréquentation.

\section{Figure 8}

Relationship between "perceived" congestion and actual density.

La CPUE diffère fortement selon les rivières (Annexe I). Pour le saumon de printemps, la CPUE est de 0,025 pour le Douron alors qu'elle n'est que de 0,002 pour le Couesnon. L'Aber Wrac'h, l'Isole, le Léguer, la Penzé, la Sée et la Sélune ont une CPUE de printemps supérieure à la moyenne et proche de 0,020. Pour les castillons, l'Aven a une CPUE de 0,063 . II ne faut donc en moyenne que 16 sorties pour y capturer un castillon. En revanche, l'Aulne a une CPUE de 0,013 pour les castillons. Notons qu'en termes de CPUE pour les castillons, les meilleures rivières sont l'Aven, le Blavet, le Trieux, la Sée, le Steïr et le Douron.

Nous n'avons pas pu mettre en évidence de lien entre la congestion des parcours de pêche et la CPUE. Nous pensons que ceci est dû au relatif désintérêt des pêcheurs pour des captures abondantes. Le pêcheur de saumon ne semble pas percevoir les autres pêcheurs comme des rivaux pour une capture, mais plutôt comme des agents perturbant la qualité de son loisir.

\section{RECONSTITUTION DE L'EFFORT TOTAL DE PÊCHE AU SAUMON DANS LE MASSIF ARMORICAIN POUR LA SAISON 2002}

Pour reconstituer les efforts de pêche totaux sur les différentes rivières de l'Ouest armoricain, nous avons utilisé les efforts constatés dans notre échantillon, ramenés à la participation des pêcheurs par département.

Pour chaque rivière, nous avons calculé, à partir de notre échantillon, le nombre de pêcheurs $\left(n_{i j}\right)$ du département $i$ fréquentant la rivière $j$. En notant $N_{i}$ le nombre total 
de pêcheurs du département $\mathrm{i}$ dans notre échantillon, nous en avons déduit un taux $\theta_{i j}$ départemental de fréquentation pour chaque rivière $\mathrm{j}$ tel que $\theta_{\mathrm{ij}}=\mathrm{n}_{\mathrm{ij}} / \mathrm{N}_{\mathrm{i}}$. A partir des effectifs départementaux $\left(E_{i}\right)$ réels de pêcheurs issus du tableau I, nous avons calculé un nombre total de pêcheurs $\left(\mathrm{N}_{\mathrm{ij}}\right)$ originaires du département $\mathrm{i}$ et fréquentant la rivière j égal à $E_{i}^{*} \theta_{i j}$. Enfin, nous en tirons le nombre total de visites $\left(V_{j}\right)$, en tenant compte des disparités départementales. Pour cela, nous avons déterminé, sur notre échantillon, le nombre moyen $\left(v_{i j}\right)$ de visites effectuées par les pêcheurs du département i sur la rivière j. Nous avons également calculé un nombre moyen $v_{\text {ik }}$ de visites d'un pêcheur du département i sur une rivière du département $\mathrm{k}$. Nous avons tenu compte des points potentiellement aberrants en calculant le nombre total $\left(V_{j}\right)$ de visites sur la rivière $j$ en retenant la règle suivante ${ }^{11}$ :

$$
v_{j}=\sum\left(E_{i} \times \frac{n_{i j}}{N_{i}} \times v_{i j}\right)=\sum^{i} N_{i j} \times v_{i j} \quad\left\{\begin{array}{l}
v_{i j}=v_{i j}, \forall n_{i j} \geq 20 \\
v_{i j}=v_{i k}, \forall n_{i j}<20
\end{array}\right.
$$

Au total, trois agrégations ont été effectuées à partir des visites totales, de printemps et d'automne. Les résultats de ces estimations sont présentés dans le tableau IV. L'effort de pêche sur les rivières de l'Ouest armoricain est estimé à 94000 visites environ pour la saison 2002. La Sée est la rivière la plus fréquentée avec près de 23000 visites par an, réparties de manière équitable entre la saison de printemps et la saison d'automne.

\section{DISCUSSION}

Dans cette partie, nous confrontons nos résultats à ceux obtenus dans d'autres études. Cela nous permet de mettre en perspective nos résultats et d'en apprécier la validité.

\section{La saison de pêche 2002 en perspective}

En termes de captures, l'année 2002 a été une mauvaise année pour la Bretagne. On constate que depuis quelques années, les captures de saumons diminuent dans tous les départements bretons (Figure 9). La Manche suit une évolution opposée puisque le niveau des captures a doublé sur la période 1997-2002 (CSP, 2003). L'année 2002 confirme les évolutions des années précédentes en termes de captures: baisse généralisée en Bretagne et augmentation dans la Manche. Depuis l'instauration de la taxe "salmonidés migrateurs " en 2002, le nombre de pêcheurs de saumon reste stable (Figure 10), et l'année 2002 est une année moyenne du point de vue du nombre de pêcheurs.

Ainsi, la saison 2002 est comparable aux années précédentes en termes d'effort de pêche. Cependant, le nombre de captures étant particulièrement faible en Bretagne en 2002, nous nous attendons à une plus faible CPUE pour ces pêcheurs que pour les années précédentes. En revanche, on s'attend à l'inverse pour les pêcheurs de la Manche.

\section{Les données issues des carnets de pêche du Conseil Supérieur de la Pêche}

Nous disposons des données recensées par le CSP dans les carnets de pêche tenus par des pêcheurs volontaires (Tableau V) sur la période 1995-97. Sur cette période, le nombre moyen de sorties par pêcheur consignées dans les carnets est proche de 40. Cette statistique est proche de nos résultats d'enquête. Nos données montrent qu'en 2002 les pêcheurs ont été moins efficaces que sur la période 1995-1997. Ce résultat est conforme à nos attentes. On remarque d'ailleurs qu'en 1995 et 1996, les captures

\footnotetext{
${ }^{11}$ Le nombre de 20 pêcheurs a été retenu en cohérence avec les données de l'échantillon. Cette limite nous a paru assez élevée pour éliminer les points aberrants et assez petite pour conserver l'hétérogénéité observée.
} 


\section{Tableau IV}

Estimation de l'effort de pêche (en nombre de visites) sur les rivières de l'Ouest armoricain en 2002.

Table IV

Estimation of total fishing effort (in number of visits) on the rivers of Western France for year 2002.

\begin{tabular}{|c|c|c|c|}
\hline $\begin{array}{l}\text { Rivière } \\
\text { River }\end{array}$ & $\begin{array}{l}\text { Saison entière } \\
\text { Whole season }\end{array}$ & $\begin{array}{l}\text { Saumons de printemps } \\
\text { Spring salmons }\end{array}$ & $\begin{array}{c}\text { Castillons } \\
\text { Grilse }\end{array}$ \\
\hline Aber Wrac'h & 436 & 310 & 126 \\
\hline Aulne & 8738 & 5125 & 3614 \\
\hline Aven & 2426 & 1285 & 1141 \\
\hline Blavet & 6066 & 3214 & 2852 \\
\hline Camfrout & 483 & 399 & 84 \\
\hline Couesnon & 1558 & 996 & 563 \\
\hline Douron & 1405 & 872 & 533 \\
\hline Ellé & 7266 & 4786 & 2479 \\
\hline Elorn & 4958 & 2889 & 2069 \\
\hline Gouet & 96 & 53 & 43 \\
\hline Goyen & 1945 & 1180 & 766 \\
\hline Isole & 679 & 410 & 268 \\
\hline Jaudy & 427 & 210 & 216 \\
\hline Jet & 1256 & 712 & 544 \\
\hline Kergroix & 469 & 303 & 166 \\
\hline Laïta & 493 & 325 & 167 \\
\hline Leff & 1252 & 863 & 389 \\
\hline Léguer & 7886 & 4615 & 3271 \\
\hline Mignonne & 739 & 487 & 251 \\
\hline Odet & 1480 & 1141 & 340 \\
\hline Penzé & 894 & 521 & 373 \\
\hline Scorff & 2086 & 1188 & 898 \\
\hline Sée & 22983 & 11548 & 11435 \\
\hline Sélune & 8258 & 4943 & 3315 \\
\hline Sienne & 4093 & 2076 & 2017 \\
\hline Steïr & 1758 & 1003 & 755 \\
\hline Trieux & 3310 & 2062 & 1248 \\
\hline Yar & 231 & 131 & 100 \\
\hline Total & 93671 & 53647 & 40023 \\
\hline
\end{tabular}

sur l'ensemble du territoire français ont été beaucoup plus nombreuses qu'en 2002. Les données CSP pour l'année 1997, comparables à celles de l'année 2002 en termes de captures, sont assez proches de nos données. Nous remarquons tout de même que le taux de bredouilles est particulièrement élevé dans notre échantillon. Deux hypothèses peuvent être émises pour expliquer les différences entre nos résultats et ceux du CSP :

- L'échantillon de pêcheurs volontaires du CSP est biaisé. Les pêcheurs volontaires ne sont pas représentatifs et sont plus efficaces que la moyenne. 


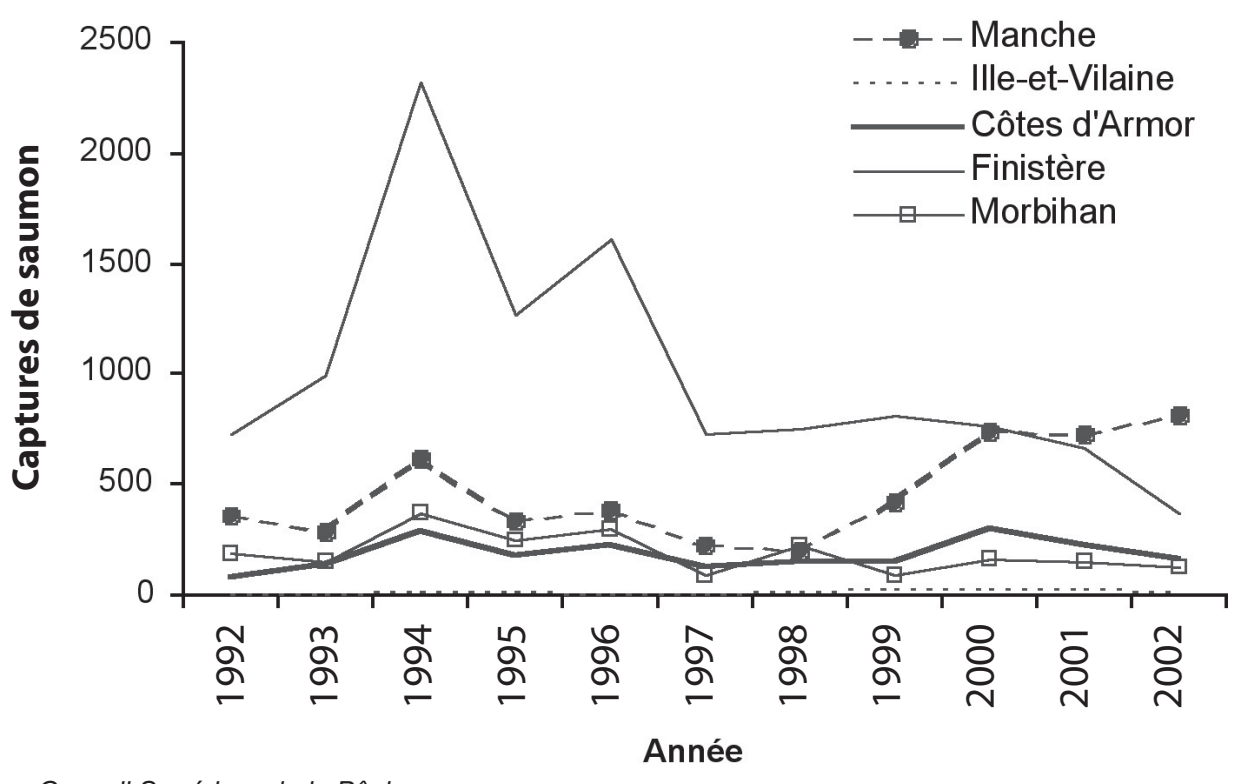

Source : Conseil Supérieur de la Pêche.

Figure 9

Évolution du nombre de captures de saumon par département depuis 1992.

Figure 9

Evolution of the number of salmon catches per county since 1992.

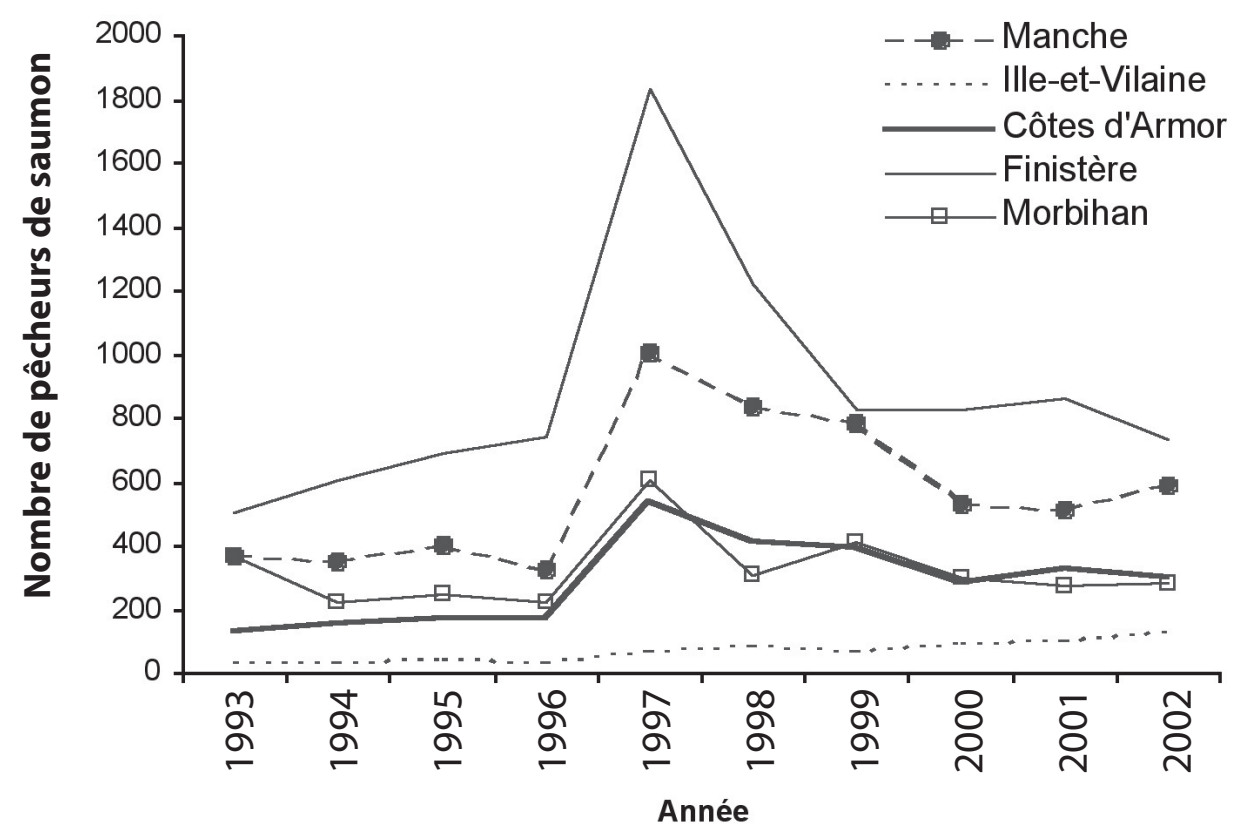

Source : Conseil Supérieur de la Pêche.

Figure 10

Évolution du nombre de pêcheurs de saumon par département depuis 1993.

Figure 10

Evolution of the number of salmon anglers per county since 1993. 
- Nos résultats d'enquête sont biaisés. Notamment, les pêcheurs ont sous-déclaré leurs captures ou surestimé le nombre de visites qu'ils font. Le problème de la sousdéclaration des captures pour la pêche au saumon est récurrent. Le CSP ajuste d'ailleurs ses statistiques. Le taux de déclaration retenu chaque année par le CSP est de l'ordre de $60 \%$ (CSP, 1996).

\section{Tableau V}

Données des carnets de pêche du CSP sur la période 1995-1997.

\section{Table V}

Data from angling diaries from CSP over the 1995-1997 period.

\begin{tabular}{|l|c|c|c|c|}
\hline \multirow{2}{*}{$\begin{array}{l}\text { Données } \\
\text { Data }\end{array}$} & \multicolumn{3}{c|}{$\begin{array}{c}\text { Enquêtes halieutiques } \\
\text { Angling surveys }\end{array}$} & $\begin{array}{c}\text { Cette enquête } \\
\text { This survey }\end{array}$ \\
\cline { 2 - 6 } & 1995 & 1996 & $\mathbf{1 9 9 7}$ & $\mathbf{2 0 0 2}$ \\
\hline $\begin{array}{l}\text { Nombre de pêcheurs } \\
\text { Number of anglers }\end{array}$ & 64 & 77 & 65 & 828 \\
\hline $\begin{array}{l}\text { Nombre moyen de sorties par pêcheur } \\
\text { Average number of visits per year per angler }\end{array}$ & 40 & 40 & 33 & 42,44 \\
\hline CPUE & 0,056 & 0,058 & 0,032 & 0,025 \\
\hline $\begin{array}{l}\text { \% de pêcheurs bredouilles } \\
\% \text { of anglers who made no catches }\end{array}$ & 38 & 32 & 46 & 67 \\
\hline $\begin{array}{l}\text { Nombre de captures totales en France } \\
\text { Total number of salmon catches in France }\end{array}$ & 2248 & 2982 & 1430 & 1746 \\
\hline
\end{tabular}

Sources : CSP (1996 à 1998).

Les résultats obtenus par l'étude des carnets de pêche ou à partir de nos résultats d'enquête sont semblables en termes d'effort de pêche mais diffèrent légèrement en termes d'efficacité. Cette différence s'observe particulièrement au niveau du taux de bredouilles.

\section{Le suivi halieutique de la pêcherie du Scorff (Morbihan)}

Le tableau VI reprend les données de suivi halieutique de la pêcherie de saumon du Scorff sur la période 1992-2001. Ce suivi est composé de carnets de pêche, remplis par une quinzaine de pêcheurs volontaires, et d'une enquête sur site servant à estimer le nombre total de pêcheurs. Sur cette période, on constate une baisse du nombre annuel moyen de visites et de l'effort total sur le Scorff. Le nombre de visites annuelles s'établit autour de la vingtaine pour les trois dernières années du suivi halieutique. II est du même ordre de grandeur que nos résultats. En revanche, l'effort total de pêche s'établit à 1500 visites environ. Nos résultats montrent un effort nettement supérieur, proche de 2 000, semblable à l'effort de pêche observé sur le Scorff pour la période 1994-1997.

Les analyses de CPUE obtenues par suivi halieutique confirment nos résultats d'enquête et montrent des CPUE proches de 0,010 pour les saumons de printemps, et de 0,040 pour les castillons.

Les résultats de notre enquête sont semblables à ceux obtenus à partir d'une méthodologie différente (suivi sur site) sur le Scorff. Nous remarquons tout de même que l'effort total que nous estimons diffère nettement des résultats obtenus par PREVOST (1993, 1995, 1996, 1998, 1999, 2000, 2001, 2002). II est possible que notre estimation traduise un transfert de pêcheurs des rivières du sud Finistère (Aven, Ellé et rivières de Quimper) vers le Scorff, à la suite des faibles captures enregistrées sur ces rivières en 2001 et 2002. 


\section{Tableau VI}

Données du suivi halieutique de la pêcherie du Scorff sur la période 1992-2001.

Table VI

Data from angling surveys on the Scorff River over the 1992-2001 period.

\begin{tabular}{|c|c|c|c|c|c|c|}
\hline \multirow[b]{2}{*}{$\begin{array}{c}\text { Année } \\
\text { Year }\end{array}$} & \multirow{2}{*}{$\begin{array}{l}\text { Nombre de } \\
\text { pêcheurs } \\
\text { Number of } \\
\text { anglers }\end{array}$} & \multirow{2}{*}{$\begin{array}{l}\text { Sorties par } \\
\text { pêcheur et par an } \\
\text { Visits per angler } \\
\text { and per year }\end{array}$} & \multirow{2}{*}{$\begin{array}{c}\text { Effort annuel } \\
\text { total } \\
\text { Total annual } \\
\text { effort }\end{array}$} & \multicolumn{3}{|c|}{ CPUE } \\
\hline & & & & $\begin{array}{c}\text { Globale } \\
\text { Whole } \\
\text { year }\end{array}$ & $\begin{array}{l}\text { Printemps } \\
\text { Spring } \\
\text { salmon }\end{array}$ & $\begin{array}{c}\text { Castillons } \\
\text { Grilse }\end{array}$ \\
\hline 1992 & 9 & 36,0 & 1147 & 0,012 & - & - \\
\hline 1994 & 18 & 44,4 & 2026 & 0,031 & $0,014^{*}$ & $0,040^{\star \star}$ \\
\hline 1995 & 18 & 39,9 & 2092 & 0,041 & $0,017^{*}$ & $0,111^{\star *}$ \\
\hline 1997 & 30 & 31,1 & 2547 & 0,017 & $0,005^{*}$ & $0,036^{\star \star}$ \\
\hline 1998 & 24 & 30,2 & 1837 & 0,041 & $0,004^{*}$ & $0,087^{\star *}$ \\
\hline 1999 & 17 & 24,8 & 1766 & 0,018 & $0,006^{*}$ & $0,043^{\star \star}$ \\
\hline 2000 & 17 & 19,8 & 1497 & 0,034 & $0,011^{*}$ & $0,077^{\star *}$ \\
\hline 2001 & 16 & 22,4 & 1383 & 0,027 & $0,008^{*}$ & $0,040^{\star *}$ \\
\hline \multicolumn{7}{|c|}{ Cette enquête / This survey } \\
\hline 2002 & 51 & 16,5 & 2086 & 0,017 & 0,006 & 0,031 \\
\hline
\end{tabular}

Sources : PREVOST (1993 à 2002).

* CPUE calculée sur les trois premiers mois de pêche. ** CPUE calculée sur la période de prolongation.

\section{Les enquêtes halieutiques sur le Blavet (Morbihan)}

Ces enquêtes effectuées entre 1997 et 1998 présentent des résultats qui diffèrent des nôtres (Tableau VII), surtout au niveau de l'agrégation du nombre de visites. L'effort total estimé sur le Blavet est compris entre 1000 et 1500 visites. Nous estimons ce même effort à 6066 visites pour la saison 2002.

Nous pensons que cette énorme différence s'explique par une forte sous-estimation de l'effort de pêche sur le Blavet pour les années 1997 et 1998, et une conjoncture halieutique très favorable à cette rivière pour la saison 2002 .

La sous-estimation de l'effort total de pêche est liée à l'utilisation d'une mauvaise technique d'agrégation des visites individuelles. En effet, l'enquête halieutique de 1997 sur le Blavet repose sur un échantillon de 112 pêcheurs obtenu par 52 recensements sur site. Notons tout d'abord que le nombre réel de pêcheurs ayant fréquenté le Blavet pendant la saison de pêche 1997 doit être supérieur à ce nombre. L'évaluation de l'effort total de pêche sur le Blavet est déduite de la répartition des pêcheurs en classes (obtenues par les fréquences de comptage lors des recensements) et de l'attribution d'un nombre arbitraire de visites. Ainsi, les auteurs remarquent qu'ils ont enquêté 112 pêcheurs dont 60 ont été comptés une fois, 41 de 2 à 5 fois et 11 plus de 5 fois. Ils évaluent l'effort individuel des pêcheurs de ces trois classes à moins de 5 sorties, une dizaine de sorties et une centaine de sorties respectivement. Ils en déduisent un effort de pêche total compris entre 1000 et 1500 visites par an, qui correspond à un effort de pêche individuel compris entre 9 et 13 sorties par pêcheur et par an. Toutes les enquêtes menées précédemment montrent que l'effort de pêche individuel moyen pour un pêcheur de saumon est proche de 40 sorties par an (cf. paragraphes précédents). En estimant le nombre de pêcheurs à 120 et en retenant cette valeur pour l'effort individuel moyen, l'effort total de pêche sur le Blavet pour la saison 1997 peut être estimé à 4800 visites, nombre plus proche de notre estimation pour la saison 2002. 
De plus, la saison 2002 a été particulièrement riche en captures sur le Blavet par comparaison avec les autres rivières. Le CSP estime à 122 le nombre de captures de saumon sur le Blavet, ce qui en fait la meilleure rivière bretonne pour les captures, devant le Léguer (96 captures), les rivières de Quimper (80 captures), le bassin ElléIsole (63 captures) et l'Aulne (52 captures). En 1997, le Blavet n'arrive qu'en septième position, avec 45 captures, derrière l'Aulne (424 captures), l'Ellé (86 captures), le Léguer (64 captures), l'Elorn (63 captures), le Douron (51 captures), le Trieux (49 captures). Comparativement aux autres rivières, les captures ont été très élevées sur le Blavet en 2002. Cette année-là, le Blavet représente $18,5 \%$ des captures de saumon en Bretagne contre 4,8 \% en 1997 et 10,6 \% en 1998. Nous pensons donc qu'il est normal d'observer un effort total de pêche plus élevé sur le Blavet en 2002 que pour les années 1997 et 1998.

\section{Tableau VII}

Données des enquêtes halieutiques sur le Blavet pour les années 1997 et 1998.

\section{Table VII}

Data from salmon angling surveys on the Blavet river for years 1997 and 1998.

\begin{tabular}{|l|c|c|c|}
\hline \multirow{2}{*}{\multicolumn{1}{|c|}{$\begin{array}{c}\text { Données } \\
\text { Data }\end{array}$}} & \multicolumn{2}{c|}{$\begin{array}{c}\text { Enquêtes halieutiques } \\
\text { Angling surveys }\end{array}$} & $\begin{array}{c}\text { Cette enquête } \\
\text { This survey }\end{array}$ \\
\cline { 2 - 4 } & 1997 & $\mathbf{1 9 9 8}$ & $\mathbf{2 0 0 2}$ \\
\hline $\begin{array}{l}\text { Nombre de pêcheurs } \\
\text { Number of anglers }\end{array}$ & 112 & 85 & 76 \\
\hline CPUE & 0,04 à 0,05 & 0,05 à 0,08 & 0,034 \\
\hline $\begin{array}{l}\text { Estimation de l'effort total } \\
\text { Total fishing effort estimation }\end{array}$ & $\begin{array}{r}1000-1500 \text { visites } \\
\text { par an }\end{array}$ & 6066 visites \\
\hline $\begin{array}{l}\text { Age moyen du pêcheur } \\
\text { Average age of anglers }\end{array}$ & 44 ans & 44 ans & 52 ans \\
\hline $\begin{array}{l}\text { Proportion d'ouvriers et employés dans } \\
\text { l'échantillon } \\
\text { Proportion of blue- and white- collar } \\
\text { workers in the sample }\end{array}$ & $42 \%$ & $42 \%$ & $40,7 \%$ \\
\hline $\begin{array}{l}\text { Proportion de retraités dans l'échantillon } \\
\text { Proportion of retired persons in the sample }\end{array}$ & $25 \%$ & $25 \%$ & $30,3 \%$ \\
\hline
\end{tabular}

Sources : Fédération du Morbihan pour la Pêche et la Protection des Milieux Aquatiques (1998 et 1999).

Pour la structure de la population de pêcheurs, on remarque que nos données d'enquête sont semblables à celles obtenues en 1997 et 1998. Toutefois, il semble que la population de pêcheurs de saumon du Blavet ait légèrement vieilli, ce qui se traduit par un âge moyen plus élevé et une plus forte proportion de retraités parmi les pêcheurs.

Finalement, les résultats d'enquête pour les saisons de pêche au saumon 1997 et 1998 sont similaires à nos observations, les différences observées s'expliquant principalement par la conjoncture halieutique, très favorable au Blavet pour la saison 2002.

\section{L'analyse économique des pêcheries de la Sée, de la Sélune et de l'Elorn}

Les données publiées par BONNIEUX et VERMERSCH (1993) et BONNIEUX (2000) présentent l'analyse économique des résultats d'une enquête menée sur site pendant la saison de pêche 1990 sur l'Elorn, la Sée et la Sélune. Nous en synthétisons certaines données dans le tableau VIII. Leur échantillon est de taille comparable au nôtre. 
Les données socio-démographiques de notre échantillon sont semblables à celles de l'enquête de 1990. L'âge, l'expérience de pêche et le nombre de retraités sont cohérents entre les deux sources de données, indiquant toutefois un probable vieillissement de la population de pêcheurs lors des dix dernières années. Par contre, les données d'effort peuvent différer assez nettement. Celles relatives aux captures (nombre de captures par an et pourcentage de pêcheurs bredouilles) restent comparables dans la mesure où elles doivent être interprétées à la lumière des contextes halieutiques des années de référence. De même, les distances parcourues par les pêcheurs restent comparables.

En revanche, et c'est particulièrement net sur l'Elorn, les pêcheurs d'aujourd'hui semblent fréquenter moins de sites substituts. La forte différence observée pour l'Elorn peut éventuellement s'expliquer par la gestion particulière de cette rivière qui ne fait partie d'aucun groupement réciprocitaire, ce qui inciterait les pêcheurs qui la pêchent à moins fréquenter d'autres rivières. Finalement, les données relatives au nombre de visites nous laissent perplexes. Nos données sont nettement inférieures à celles de BONNIEUX et VERMERSCH (1993), sans que nous ayons d'explication à cette différence, hormis l'hypothèse d'un changement de comportement des pêcheurs lors de la dernière décennie.

\section{Tableau VIII}

Données de l'étude économique des pêcheries de la Sée, de la Sélune et de I'Elorn.

\section{Table VIII}

Data from the economic analysis of recreational fishing on the Sée, Sélune and Elorn Rivers.

\begin{tabular}{|c|c|c|c|c|c|}
\hline \multirow{3}{*}{$\begin{array}{l}\text { Données } \\
\text { Data }\end{array}$} & \multirow{2}{*}{\multicolumn{2}{|c|}{$\begin{array}{c}\begin{array}{c}\text { Bonnieux et } \\
\text { Vermersch }\end{array} \\
1990 \\
\end{array}$}} & \multicolumn{3}{|c|}{$\begin{array}{l}\text { Cette étude } \\
\text { This survey }\end{array}$} \\
\hline & & & \multicolumn{3}{|c|}{2002} \\
\hline & Elorn & $\begin{array}{l}\text { Sée- } \\
\text { Sélune }\end{array}$ & Elorn & Sée & Sélune \\
\hline \begin{tabular}{|l|} 
Nombre de pêcheurs \\
Number of anglers
\end{tabular} & 72 & 101 & 55 & 187 & 150 \\
\hline $\begin{array}{l}\text { Nombre moyen de visites } \\
\text { Average number of visits }\end{array}$ & 62 & 47 & 37,0 & 39,0 & 18,5 \\
\hline \begin{tabular}{|l|} 
Distance au site de pêche $(\mathrm{km})$ \\
Distance to fishing site $(\mathrm{km})$ \\
\end{tabular} & 76 & 85 & 110,8 & 39,9 & 59,1 \\
\hline $\begin{array}{l}\text { Age moyen } \\
\text { Average Age } \\
\end{array}$ & 41,8 & 46,8 & 49,6 & 50,9 & 50,4 \\
\hline $\begin{array}{l}\text { Expérience (en années) } \\
\text { Experience (in years) }\end{array}$ & 19 & 15 & 18,5 & 15,6 & 15,6 \\
\hline $\begin{array}{l}\text { \% de pêcheurs retraités } \\
\% \text { of retired anglers }\end{array}$ & 14 & 24 & 23,6 & 35,8 & 34,0 \\
\hline $\begin{array}{l}\text { \% de pêcheurs fréquentant un site substitut } \\
\% \text { of anglers visiting a substitute site }\end{array}$ & \multicolumn{2}{|c|}{63} & 14,5 & 48,1 & 72,7 \\
\hline $\begin{array}{l}\text { Captures par an } \\
\text { Catches per year }\end{array}$ & 2,4 & 1,3 & 0,7 & 1,4 & 0,4 \\
\hline $\begin{array}{l}\% \text { de pêcheurs bredouilles } \\
\% \text { of anglers who made no catches }\end{array}$ & \multicolumn{2}{|c|}{48} & 78,2 & 47,6 & 76 \\
\hline
\end{tabular}

Source : BONNIEUX et VERMERSCH (1993). 
Deux autres études socio-économiques offrent un élément de comparaison avec nos données. Les pêcheurs interrogés par DESAIGUES, LESGARDS et LISCIA (1994) sont relativement âgés (46 ans) et font 17,5 sorties par an pour une distance moyenne de $22 \mathrm{~km}$. Ces données, légèrement inférieures aux nôtres, ne concernent pas exclusivement des pêcheurs de salmonidés, et en particulier de salmonidés migrateurs, dont nous savons qu'ils sont particulièrement avides de sorties de pêche, souvent au détriment des autres formes de pêche et de loisirs (BONNIEUX, 2000). D'ailleurs, pour un échantillon constitué majoritairement de pêcheurs de salmonidés et de carnassiers, ARMAND, BONNIEUX et CHANGEUX (2002), trouvent un effort de pêche de 46 sorties par an, plus élevé que DESAIGUES, LESGARDS et LISCIA (1994) et très proche de nos résultats. Sur la pêcherie de salmonidés du Lignon du Velay, BONNIEUX, GUERRIER et FOUET (2002) trouvent un effort de pêche de 16,9 visites par an mais il s'agit là d'une mesure d'effort par pêcheur et par rivière. La même mesure à l'échelle de notre échantillon est de 27 sorties par an.

\section{CONCLUSION}

Nous avons mis en place une enquête téléphonique auprès des pêcheurs de saumon qui fréquentent les rivières de Bretagne et de la Manche. L'échantillon de 827 individus ainsi obtenu permet de caractériser l'effort individuel de pêche, l'effort par rivière et la structure de la population de pêcheurs. Nous estimons avoir enquêté près de $44 \%$ de la population de pêcheurs concernés.

Les résultats de notre étude montrent que la situation des rivières de l'Ouest armoricain est très hétérogène. En particulier, on constate que certaines rivières sont très fréquentées: II s'agit des plus grosses et des plus poissonneuses comme la Sélune, la Sée, l'Aulne, l'Ellé, le Léguer et le Blavet. En revanche, les petits abers, comme le Kergroix, le Camfrout, le Yar ou la Penzé sont peu fréquentés. Nous mettons en évidence des problèmes de congestion pour les rivières les plus importantes comme la Sélune, la Sée, le Trieux et le Léguer. Ce point, qui n'est pas évoqué dans d'autres études, peut intéresser les gestionnaires de la pêche de loisir.

Nous montrons également que l'effort individuel de pêche, bien que très variable, est, en moyenne, proche de 40 sorties par an. En moyenne, un pêcheur parcourt $28 \mathrm{~km}$ pour se rendre sur une rivière. Ceux qui sont les plus proches des sites de pêche pratiquent plus souvent leur loisir. Les pêcheurs visitent généralement plusieurs rivières (2 en moyenne). L'efficacité des pêcheurs semble dépendre de leur expérience, mais également de l'appât utilisé. Les pêcheurs de saumon pratiquent généralement d'autres pêches, telles que la pêche en eaux vives, la pêche à la truite de rivière et la pêche en mer. Enfin, près d'un individu enquêté sur trois a été pêcher à l'étranger lors des trois dernières années. Ces séjours sont effectués principalement dans les lles britanniques et en Europe du Nord, pour pêcher le saumon.

Notre étude permet de caractériser la population de pêcheurs de saumon de l'Ouest armoricain. Nous montrons qu'il s'agit d'une population fortement masculine, âgée de 52 ans en moyenne. La plupart des pêcheurs de saumon sont retraités, ouvriers ou employés. Leur passion pour la pêche et la recherche du calme et de la solitude sont leurs motivations principales pour la pratique de ce loisir. Les captures ne représentent qu'un intérêt secondaire pour la majorité des pêcheurs. Ils pensent aussi que les pollutions agricoles, les captures de saumon faites par les professionnels et les atteintes physiques aux milieux aquatiques (canalisation) ont des effets nuisibles sur la qualité de leur loisir. Nous montrons également que les mesures de préservation des stocks de saumon (TAC, repeuplements et parcours "no-kill ») sont perçues comme bénéfiques et susceptibles d'améliorer la qualité de la pêche. En revanche, les règlements qui induisent une limitation de l'effort de pêche sont plus mal perçus. En particulier, les pêcheurs sont favorables aux 
fermetures hebdomadaires, mais estiment que le recul de l'ouverture est une mesure plutôt néfaste. De même, les pêcheurs de saumon ont un avis mitigé sur le développement de la réciprocité nationale. Nous pensons que le recul de l'ouverture, qui réduit la durée de la saison et concentre l'effort de pêche, et les accords réciprocitaires sont vus négativement par les pêcheurs, car ils favorisent la congestion des parcours de pêche.

Cette étude nous a également permis de reconstituer l'effort de pêche total pour la saison 2002 sur les rivières de l'Ouest armoricain. L'effort annuel total de pêche est estimé à près de 93000 visites, dont $57 \%$ sont effectuées pendant la saison de printemps. A elles seules, la Sée et la Sélune supportent le tiers de cet effort total.

Enfin, nous montrons que nos résultats sont cohérents avec ceux des études existantes. Ces éléments valident les stratégies mises en œuvre pour limiter les biais d'enquête. Ainsi, il s'avère que la base de données constituée est un outil fiable et intéressant pour l'analyse de l'effort de pêche et des comportements des pêcheurs. Elle nous servira pour mener une analyse économique des bénéfices sociaux associés à la pêche de loisir pour le saumon en Bretagne et dans la Manche. Nous mettrons en relation demande et qualité de la pêche en nous intéressant particulièrement aux dégradations des milieux du fait activités humaines (agriculture, production d'hydroélectricité, navigation fluviale) et à la qualité des expériences récréatives (abondance des captures et congestion des sites).

\section{BIBLIOGRAPHIE}

ARMAND C., BONNIEUX F., CHANGEUX T., 2002. Évaluation économique des plans de gestion piscicole. Bull. Fr. Pêche Piscic., 365/366, 565-578.

BILOCQ F., 1996. Conception et évaluation de questionnaires. Actes des journées de méthodologie statistique, Collection INSEE méthodes, 69-70-71, 77-92.

BONNIEUX F., 2000. Évaluation de l'impact sur l'économie régionale de la pêche amateur : l'exemple des salmonidés migrateurs. Bull. Fr. Pêche Piscic., 357/358, 421-437.

BONNIEUX F., GUERRIER C., FOUET J.P., 2002. Valorisation économique des usages de l'eau sur le Lignon du Velay. Synthèse du rapport final, INRA-ESR, Rennes, $17 \mathrm{p}$.

BONNIEUX F., VERMERSCH D., 1993. Bénéfices et coûts de la protection de l'eau: application de l'approche contingente à la pêche sportive. Revue d'Économie Politique, 103, 83-104.

CONSEIL SUPÉRIEUR DE LA PÊCHE, 1996. Le saumon atlantique en France en 1995. - Captures par les pêcheurs amateurs et professionnels en eau douce. - Éléments de connaissance et de gestion des stocks. Centre National d'Interprétation des Captures, CSP Rennes, Cesson-Sévigné, 63 p.

CONSEIL SUPÉRIEUR DE LA PÊCHE, 1997. Le saumon atlantique en France en 1996 - Captures par les pêcheurs amateurs et professionnels en eau douce - Éléments de connaissance et de gestion des stocks. Centre National d'Interprétation des Captures, CSP Rennes, Cesson-Sévigné, 45 p.

CONSEIL SUPÉRIEUR DE LA PÊCHE, 1998. Le saumon atlantique en France en 1997 - Captures par les pêcheurs amateurs et professionnels en eau douce - Éléments de connaissance et de gestion des stocks. Centre National d'Interprétation des Captures, CSP Rennes, Cesson-Sévigné, 63 p.

CONSEIL SUPÉRIEUR DE LA PÊCHE, 2003. Le saumon en France en 2002. Centre National d'Interprétation des Captures, CSP Rennes, Cesson-Sévigné, 4 p. 
DESAIGUES B., LESGARDS V., LISCIA D., 1994. La valeur de l'eau à usage récréatif : Application aux rivières du Limousin. In : POINT P., La valeur économique des hydrosystèmes: méthodes et modèles d'évaluation des services délivrés, Économica, Paris, 13-35.

ESCOFIER B., PAGES J., 1998. Analyses factorielles simples et multiples, $3^{\mathrm{e}}$ édition, Dunod, Paris.

FÉDÉRATION DU MORBIHAN POUR LA PÊCHE ET LA PROTECTION DES MILIEUX AQUATIQUES, 1998. Enquête halieutique sur les pêcheurs de saumon du Blavet (1997). Rapport pour le contrat de plan État-Région 1994-1998 : Actions en faveurs des poissons migrateurs, $14 \mathrm{p}$.

FÉDÉRATION DU MORBIHAN POUR LA PÊCHE ET LA PROTECTION DES MILIEUX AQUATIQUES, 1999. Enquête halieutique sur les pêcheurs de saumon du Blavet (1998). Rapport pour le contrat de plan État-Région 1994-1998 : Actions en faveurs des poissons migrateurs, $14 \mathrm{p}$.

HECKMAN J.J., 1979. Sample selection bias as a specification error. Econometrica, 42(4), 153-161.

INSEE, 2001. Les tableaux de l'économie française - 2000. INSEE, Paris.

LE GOFFE P., SALANIÉ J., 2005. La gestion associative de la pêche en France: une analyse économique à partir du cas des salmonidés. Bull. Fr. Pêche Piscic., 375, 15-34.

LAMBERT T., LEBART L., MORINEAU A., PLEUVRET P., 1996. Manuel de référence de SPAD, CISIA-CERESTA, Saint-Mandé.

LYLE J.M, COLEMAN A.P.M., WEST L., CAMPBELL, D., HENRY G.W., 2002. New largescale methods for evaluating sport fisheries. In: PITCHER T.J. et HOLLINGWORTH C., Recreational fisheries: Ecological, economic and social evaluation, Fish and Aquatic Resources Series 8, Blackwell Science, Londres, 207-226.

PREVOST E., 1993. Enquête halieutique sur l'exploitation du saumon par pêche à la ligne sur dans Scorff - Bilan de l'année 1992. Rapport INRA Rennes - Unité d'Écologie Aquatique, $6 \mathrm{p}$.

PREVOST E., 1995. Suivi halieutique de l'exploitation du saumon par pêche à la ligne sur le Scorff - Bilan de l'année 1994. Rapport INRA Rennes - Unité d'Écologie Aquatique, $8 \mathrm{p}$.

PREVOST E., 1996. Suivi halieutique de l'exploitation du saumon par pêche à la ligne sur le Scorff - Bilan 1995 et synthèse 1992-1995. Rapport INRA Rennes - Unité d'Écologie Aquatique, $11 \mathrm{p}$.

PREVOST E., 1998. Suivi halieutique de l'exploitation du saumon par pêche à la ligne sur le Scorff - La saison 1997. Rapport INRA Rennes - Unité d'Écologie Aquatique, $8 \mathrm{p}$.

PREVOST E., 1999. Suivi halieutique de l'exploitation du saumon par pêche à la ligne sur le Scorff - La saison 1998. Rapport INRA Rennes - Unité d'Écologie Aquatique, $8 \mathrm{p}$.

PREVOST E., 2000. Suivi halieutique de l'exploitation du saumon par pêche à la ligne sur le Scorff - La saison 1999. Rapport INRA Rennes - Unité d'Écologie Aquatique, $8 \mathrm{p}$.

PREVOST E., 2001. Suivi halieutique de l'exploitation du saumon par pêche à la ligne sur le Scorff - La saison 2000. Rapport INRA Rennes - Unité d'Écologie Aquatique, $7 \mathrm{p}$. 
PREVOST E., 2002. Suivi halieutique de l'exploitation du saumon par pêche à la ligne sur le Scorff - La saison 2001. Rapport INRA Rennes - Unité d'Écologie Aquatique, $16 \mathrm{p}$.

PREVOST E., PORCHER J.P., 1996. Pêche au saumon dans les cours d'eau du Massif Armoricain - Fixation du nombre total de captures autorisé (TAC) par bassin. Document du GRISAM. Évaluation et gestion des stocks de poissons migrateurs, document scientifique et technique $\mathrm{n}^{\circ} 2$. 


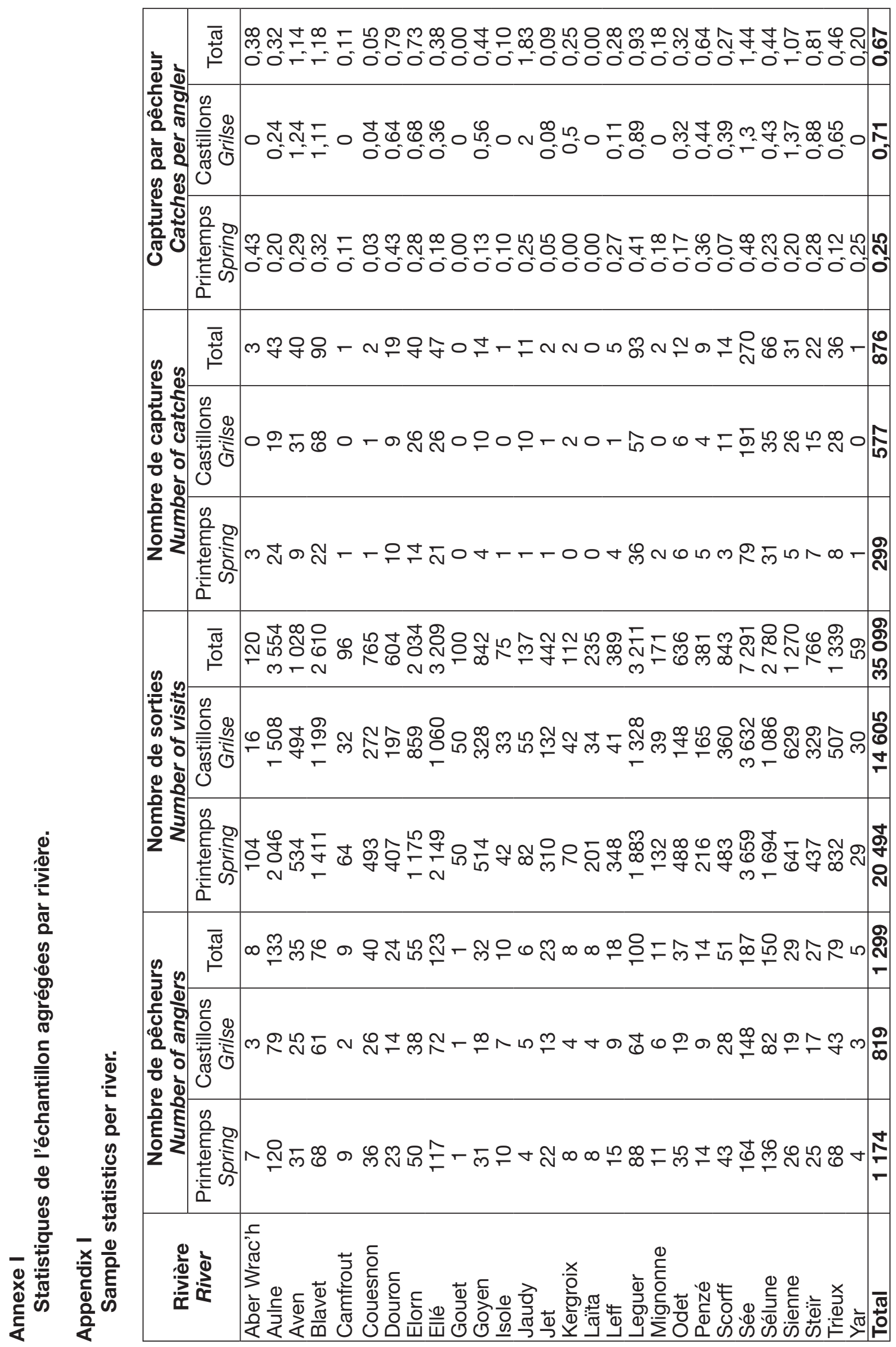




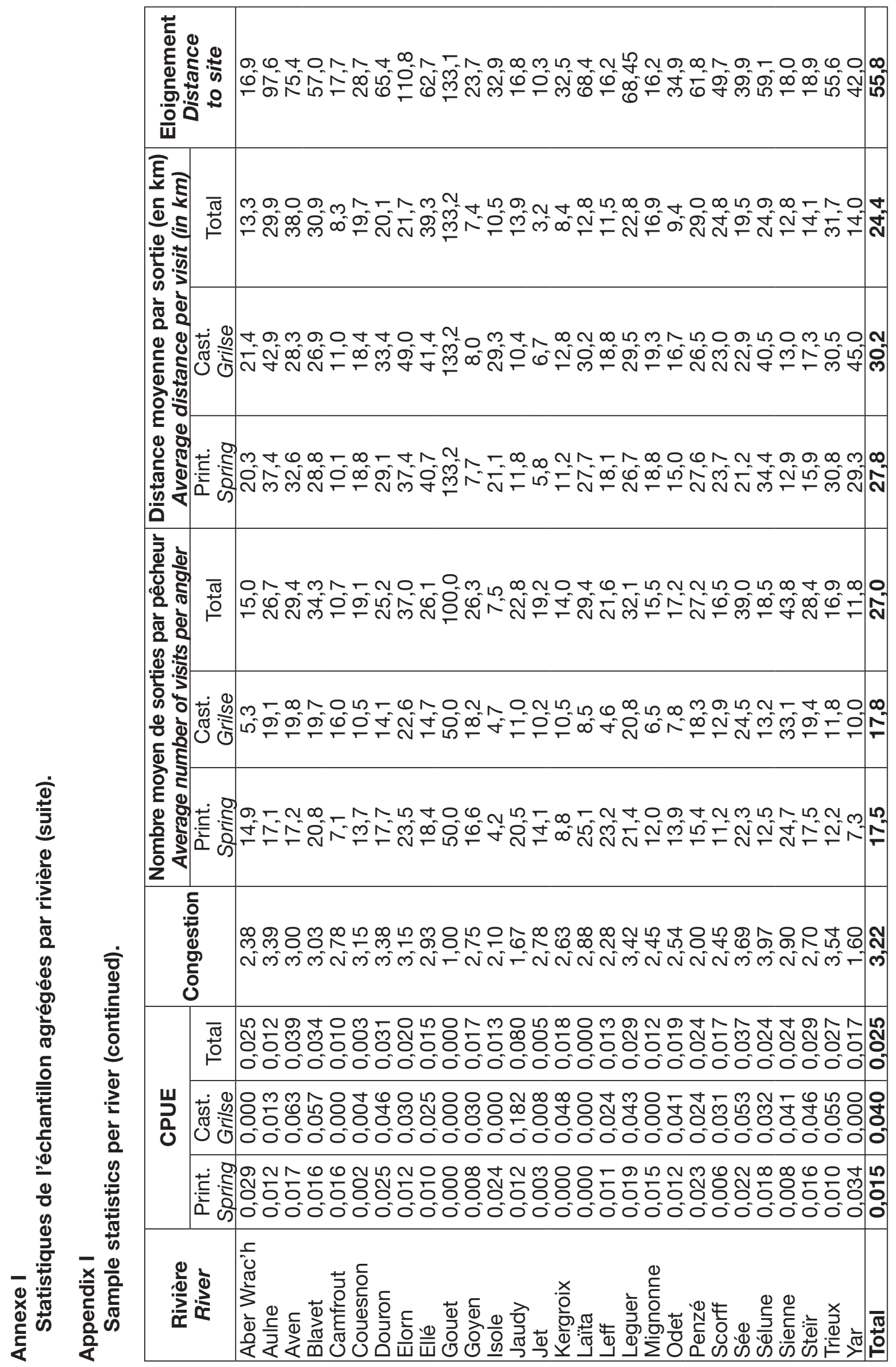


Annexe II

Détail des appréciations de congestion par rivière.

Appendix II

Assessment of congestion per river.

\begin{tabular}{|c|c|c|c|c|c|c|c|}
\hline \multirow[b]{2}{*}{$\begin{array}{c}\text { Rivière } \\
\text { River }\end{array}$} & \multicolumn{6}{|c|}{$\begin{array}{l}\text { Répartition relative des notes } \\
\text { Relative distribution of marks }\end{array}$} & \multirow[b]{2}{*}{$\begin{array}{l}\text { Effectifs } \\
\text { Numbers }\end{array}$} \\
\hline & $\begin{array}{l}\text { Très peu } \\
\text { Very little } \\
\text { (1) }\end{array}$ & $\begin{array}{c}\text { Peu } \\
\text { Little } \\
\text { (2) }\end{array}$ & $\begin{array}{c}\text { Moyen } \\
\text { Acceptable } \\
\text { (3) }\end{array}$ & $\begin{array}{c}\text { Très } \\
\text { Very } \\
\text { (4) }\end{array}$ & $\begin{array}{c}\text { Trop } \\
\text { Highly } \\
\text { (5) }\end{array}$ & $\begin{array}{c}\text { Note } \\
\text { moyenne } \\
\text { Average } \\
\text { mark }\end{array}$ & \\
\hline $\begin{array}{l}\text { Aber } \\
\text { Wrac'h }\end{array}$ & 25,0 & 25,0 & 37,5 & 12,5 & 0,0 & 2,38 & 8 \\
\hline Aulne & 1,5 & 11,3 & 41,4 & 38,3 & 7,5 & 3,39 & 133 \\
\hline Aven & 8,6 & 17,1 & 42,9 & 22,9 & 5,7 & 3,00 & 35 \\
\hline Blavet & 5,3 & 17,1 & 48,7 & 27,6 & 1,3 & 3,03 & 76 \\
\hline Camfrout & 0,0 & 44,4 & 44,4 & 0,0 & 11,1 & 2,78 & 9 \\
\hline Couesnon & 10,0 & 22,5 & 27,5 & 22,5 & 17,5 & 3,15 & 40 \\
\hline Douron & 4,2 & 16,7 & 33,3 & 29,2 & 16,7 & 3,38 & 24 \\
\hline Elorn & 5,5 & 20,0 & 38,2 & 27,3 & 9,1 & 3,15 & 55 \\
\hline Ellé & 4,9 & 23,6 & 49,6 & 17,9 & 4,1 & 2,93 & 123 \\
\hline Gouet & 100,0 & 0,0 & 0,0 & 0,0 & 0,0 & 1,00 & 1 \\
\hline Goyen & 9,4 & 21,9 & 53,1 & 15,6 & 0,0 & 2,75 & 32 \\
\hline Isole & 20,0 & 50,0 & 30,0 & 0,0 & 0,0 & 2,10 & 10 \\
\hline Jaudy & 33,3 & 66,7 & 0,0 & 0,0 & 0,0 & 1,67 & 6 \\
\hline Jet & 8,7 & 34,8 & 34,8 & 13,0 & 8,7 & 2,78 & 23 \\
\hline Kergroix & 25,0 & 12,5 & 50,0 & 0,0 & 12,5 & 2,63 & 8 \\
\hline Laïta & 12,5 & 0,0 & 75,0 & 12,5 & 0,0 & 2,88 & 8 \\
\hline Leff & 16,7 & 55,6 & 11,1 & 16,7 & 0,0 & 2,28 & 18 \\
\hline Léguer & 1,0 & 14,0 & 38,0 & 36,0 & 11,0 & 3,42 & 100 \\
\hline Mignonne & 18,2 & 27,3 & 45,5 & 9,1 & 0,0 & 2,45 & 11 \\
\hline Odet & 5,4 & 51,4 & 29,7 & 10,8 & 2,7 & 2,54 & 37 \\
\hline Penzé & 35,7 & 28,6 & 35,7 & 0,0 & 0,0 & 2,00 & 14 \\
\hline Scorff & 19,6 & 39,2 & 23,5 & 11,8 & 5,9 & 2,45 & 51 \\
\hline Sée & 4,3 & 7,5 & 25,1 & 40,1 & 22,5 & 3,69 & 187 \\
\hline Sélune & 3,3 & 6,0 & 12,0 & 48,0 & 30,7 & 3,97 & 150 \\
\hline Sienne & 10,3 & 27,6 & 34,5 & 17,2 & 10,3 & 2,90 & 29 \\
\hline Steir & 11,1 & 33,3 & 37,0 & 11,1 & 7,4 & 2,70 & 27 \\
\hline Trieux & 6,3 & 3,8 & 38,0 & 32,9 & 19,0 & 3,54 & 79 \\
\hline Yar & 60,0 & 20,0 & 20,0 & 0,0 & 0,0 & 1,60 & 5 \\
\hline
\end{tabular}


Annexe III

Distance moyenne d'une sortie (en $\mathrm{km}$ ) en fonction de la préférence pour les caractéristiques des sites de pêche.

Appendix III

Average distance of visit (in $\mathbf{k m}$ ) as a function of the preferences for fishing sites characteristics.

\begin{tabular}{|l|c|c|c|}
\hline \multicolumn{1}{|c|}{$\begin{array}{c}\text { Niveau de } \\
\text { préférence } \\
\text { Level of preference }\end{array}$} & $\begin{array}{c}\text { Site de proximité } \\
\text { Proximity site }\end{array}$ & $\begin{array}{c}\text { Rivière à caractère } \\
\text { sauvage } \\
\text { Wild looking river }\end{array}$ & $\begin{array}{c}\text { Parcours peu } \\
\text { fréquentés } \\
\text { Low congested } \\
\text { sites }\end{array}$ \\
\hline $\begin{array}{l}\text { Inimportant } \\
\text { Unimportant }\end{array}$ & 35,6 & 15,2 & 21,4 \\
\hline $\begin{array}{l}\text { Peu important } \\
\text { Somewhat Important }\end{array}$ & 31,3 & 28,9 & 33,6 \\
\hline Important & 23,3 & 22,8 & 29,6 \\
\hline $\begin{array}{l}\text { Très important } \\
\text { Very important }\end{array}$ & 25,5 & 31,5 & 22,9 \\
\hline $\begin{array}{l}\text { Décisif } \\
\text { Crucial }\end{array}$ & 16,4 & 30,8 & 27,3 \\
\hline
\end{tabular}

